\title{
A REVIEW OF ANALYTICAL TECHNIQUES FOR DETERMINATION OF ANTI-HIV DRUGS
}

\author{
RAJEEV KUMAR MISHRA ${ }^{1}$, NEELESH CHAUBEY ${ }^{1}$, JAY RAM PATEL ${ }^{2}$, SATISH MISHRA ${ }^{3}$, ROHIT SINGH ${ }^{4}$
}

${ }^{1}$ Sri Satya Sai University of Technology and Medical Science sehore M. P. India, ${ }^{1}$ Dean Pharmacy, Sri Satya Sai University of Technology and

Medical Science Sehore M. P., ${ }^{2}$ Rungta Institute of Pharmaceutical Science and Research, Bhilai Durg C. G. India, ${ }^{3}$ Gulabkali Memorial College of Pharmacy Chakghat Rewa M. P India, ${ }^{4}$ Aditya College of Pharmacy Satna M. P. India

Email: rajeevrewa86@gmail.com

Received: 14 Jul 2020, Revised and Accepted: 23 Sep 2020

\begin{abstract}
Pharmaceutical analysis plays a very prominent role in quality assurance as well as quality control of bulk drugs and pharmaceutical formulations. Rapid increase in pharmaceutical industries and production of drug in various parts of the world has brought a rise in demand for new analytical techniques in the pharmaceutical industries. As a consequence, analytical method development has become the basic activity of analysis. From the times of yore, people were trying to find safe and sound ways to treat viral infections. In the current scenario, due to the emerging of new viruses, the development of drugs for their treatment is also gaining equal importance. Before launching to the market, these drugs should undergo a validation process. High-performance liquid chromatography (HPLC) coupled with ultraviolet (UV), Photodiode array detectors (PDA), Mass spectrophotometer (MS) detectors etc. is one of the fastest, safe and precise technologies used for determination and separation of pharmaceutical drugs, impurities and biological samples. HPLC is versatile and it takes less time for quantification of drugs as compared to old liquid chromatography techniques. Tenofovir disoproxil fumarate (TDF), Emtricitabine (FTC) and Efavirenz (EFV) is antiretroviral medicine used treat AIDS as well as chronic Hepatitis-B. It is used alone or with other HIV medications to help control HIV infection. The present review article assesses the published analytical methods and a variety of approach for investigation of TDF, FTC and EFV in bulk drug as well as pharmaceutical formulations including combinations. The present studies revealed that HPLC technique along with the spectroscopic have been most widely explored for the analysis. The investigatory review may provide the comprehensive details to the researchers who are working in the area of analytical research of TDF, FTC and EFV.
\end{abstract}

Keywords: Pharmaceutical analysis, High-performance liquid chromatography, Tenofovir disoproxil fumarate, Emtricitabine, Efavirenz

(C) 2020 The Authors. Published by Innovare Academic Sciences Pvt Ltd. This is an open access article under the CC BY license (http://creativecommons.org/licenses/by/4.0/) DOI: http://dx.doi.org/10.22159/ijap.2020v12i6.39040. Journal homepage: https://innovareacademics.in/journals/index.php/ijap

\section{INTRODUCTION}

The main goal of the pharmaceutical industry is to provide drug products with sufficient quality, efficacy and safety. The development of a new drug product and its production consist of many pharmaceutical processes, including analytical testing. The analytical data generated support further decisions on how development should be pursued or provide information on whether a drug product should be released. It is important that each such development or production process provide credible results with constant quality and therefore, it needs to be controlled and, if necessary, continually improved. By improvement of the quality of a pharmaceutical process, the quality of a drug product is also improved. Analytical methods are among the most critical processes in drug product development and production. They play a key role in supporting other development and production processes throughout all stages of a drug product's life cycle. It is essential that an analytical method be precise, accurate and reliable, making it suitable for its intended purpose [1, 2]. In most cases, the main working principle of an analytical method is separation of the analytes present in the sample. Liquid chromatography (LC) techniques are most commonly employed, such as HPLC or ultraperformance liquid chromatography (UPLC), often in reversedphase mode with UV absorbance detection. The purposes of analysis differ depending on the number, importance and relation of analytes that are required to be determined. Analytical methods for the assay of an active pharmaceutical ingredient (API) or determination of its related substances and degradation products are most commonly applied [2]. Development of a specific and robust stability-indicating LC method for the determination of related substances and degradation products is a complex process. It requires a deliberate forced degradation of a drug substance and/or a drug product under various stress conditions, such as hydrolytic, oxidative, photolytic, or thermal conditions, to provide stressed samples containing the analyte and its degradation products. The stress conditions are more severe than the accelerated and long-term stability conditions prescribed in the ICH guidelines for stability testing. An analytical method for determination of degradation products should be capable of detecting their increase during the product's shelf life and the method for the assay should be capable of detecting any decrease in the drug substance's content during its shelf life. Such methods are stability indicating [3-6].

Recent estimates indicate that 34 million people are currently living with HIV/AIDS worldwide, with approximately 2.5 million new infections occurring annually [7]. The virus is transmitted through the exchange of virus containing fluids, including blood, breast milk, semen and genital secretions [8-10]. Routes of viral infection include sexual contact, injection drug use, from mother to child during pregnancy, childbirth, or breast-feeding, and exposure of infected body fluids to exposed membranes or tissue $[10,11]$. Antiretroviral therapy (ART) is the primary modality for the treatment and management of the disease and can substantially reduce HIV-related morbidity and mortality [12-14]. ART is strongly recommended for all HIV-infected individuals, regardless of pretreatment CD41 T cell count. Furthermore, ART has shown efficacy not only in disease management but also in viral prevention as pre-exposure prophylaxis in high-risk populations [15-18]. There are currently more than 25 antiretroviral (ARV) agents approved for HIV treatment by the U. S. Food and Drug Administration (FDA) in both single-and multi-drug formulations [19]. Combinatorial ART regimens are typically required for the sustained suppression of viral replication and clinical benefit [20]. Currently, more than 100 regimens exist for the treatment of HIV [21]. ARVs elicit their therapeutic effects through the targeted inhibition of various stages of the viral infection cycle. Thus, drug classes are stratified as CCR5 antagonists, viral fusion inhibitors, nucleoside/nucleotide reverse transcriptase inhibitors (NRTIs/NtRTIs), non-nucleoside reverse transcriptase inhibitors (NNRTIs), integrase strand transfer inhibitors (INSTIs), and protease inhibitors (PIs). Many combinatorial ART regimens incorporate drugs from more than one ARV class, and the U. S. Department of Health and Human Services (DHHS) has indicated recommended and alternative regimens for disease management [22]. In addition, new therapies are continually 
being sought that exploit new viral targets, have activity against resistant viral strains, have a lower incidence of adverse effects, and offer convenient dosing. New agents of existing classes are currently in advanced stages of clinical development [23]. The growing demand for these agents stimulates a search for new even more effective drugs, but also calls for higher level of quality control of these therapeutic substances and preparations, so that they are in the highest possible degree free from any impurities that may come from the production process, as well as from decompositions products of active or auxiliary substances. Therefore, it seems appropriate to develop new analytical methods regarding their qualitative and quantitative analysis. For this aim, different analytical methods are used for determining anti-HIV drugs. AntiHIV drugs are the recent developments of drugs and there is a great need to review the analytical work reported so far in the literatures. Efforts have been made to collect the literature from 2000 up to the present. Analytical methods allowing the determination of TDF, FTC and EFV drugs in various media, such as pharmaceutical formulations, biological matrices and environmental samples, is discussed. At present, there are five major classes of ARV drugs viz. nucleoside reverse transcriptase inhibitor [NRTI], non-nucleoside reverse transcriptase inhibitor [NNRTI], Protease inhibitors [PI], fusion inhibitor and integrase inhibitor [IIs].

The first single-tablet fixed-dose combination (FDC) antiretroviral (ARV) has been commercially available since 2006 and is marketed as Atripla $\AA$ [24]. A generic product has been commercially available in South Africa since April 2013 [25, 26] and consists of efavirenz (EFV), emtricitabine (FTC) and tenofovir disoproxil fumarate (TDF) in a ratio of $600 \mathrm{mg} / 200 \mathrm{mg} / 300 \mathrm{mg}$. TDF in this quantity is equivalent to $245 \mathrm{mg}$ tenofovir disoproxil (TNF) and $136 \mathrm{mg}$ of tenofovir [27]. The tablet is taken once daily for the treatment of HIV-1 infection [28, 29]. Once-daily FDC tablets are the simplest antiretroviral therapy available [30]. FDC ARV therapy is convenient for patients as it reduces the "pill burden" which in turn improves adherence to therapy [28-30]. FDC were initially indicated for treating HIV-positive antiretroviral naïve patients and HIV-positive pregnant women and those who are breastfeeding. It is now available to all patients on the recommendation of a physician [26]. Treatment with EFV, FTC and TNF is the preferred first-line therapy for antiretroviral naïve HIV-1-infected persons [30]. Bioequivalence between the dosage form containing a single molecule and the FDC in addition to favourable pharmacokinetics facilitates once daily dosing of EFV, FTC and TNF [24, 30].

\section{Nucleoside reverses transcriptase inhibitors (NRTIs)}

The first generation of ARV drugs is NRTIs permitted to treat HIV [31]. Reverse transcriptase is an HIV enzyme that converts viral RNA into DNA in host $\mathrm{CD}_{4}$ cells and the process is known as reverse transcription. NRTIs inhibit the enzyme reverse transcriptase and prevent the synthesis of DNA. Without reverse transcriptase, HIV cannot replicate and infection cannot spread. Nucleoside analogues possess structural similarity with the natural building blocks of DNA and have to undergo phosphorylation to become active in the body. NRTIs are falsely chosen by reverse transcriptase to build the faulty DNA that denies further addition of natural nucleotides. Thus, the new DNA built incorrectly led to halt HIV replication [32]. Following are some NRTIs used for HIV therapy: Zidovudine, Didanosine, Stavudine, Lamivudine, Abacavir, Adefovir, Emtricitabine (FTC), Tenofovir disoproxil fumarate (TDF).

\section{Emtricitabine (FTC)}

FTC is a synthetic fluoro derivative of thiacytidine with potent antiviral activity approved in 2003. Chemically it is a 4-amino-5fluoro-1-[[2R, 5S]-2-[hydroxymethyl]-1, 3-oxathiolan-5-yl] pyrimidin-2-one, the solubility of which in water is $112 \mathrm{mg} / \mathrm{ml}$ with $\operatorname{logP}$ value of-1.4. FTC is a white to off white, crystalline powder [33, 35]. FTC has an empirical formula of $\mathrm{C}_{8} \mathrm{H}_{10} \mathrm{FN}_{3} \mathrm{O}_{3} \mathrm{~S}$ and a relative molecular mass of $247.2 \mathrm{~g} / \mathrm{mol}[34,36]$. FTC contains no less than 99.0 percent and not more than 101.0 percent of emtricitabine $\left(\mathrm{C}_{8} \mathrm{H}_{10} \mathrm{FN}_{3} \mathrm{O}_{3} \mathrm{~S}\right)$, calculated with reference to the anhydrous reference material [34]. FTC, when combined with TDF, has shown together greater HIV RNA suppression compared to the combination of Zidovudine and Lamivudine $[37,38]$. Co administration of FTC/TDF with antiviral drugs that eliminate through kidney by means of active tubular discharge may enhance plasma TDF or FTC concentrations and/or those of simultaneously given drugs [39]. FTC undergoes phosphorylation to form active FTC triphosphates metabolite using cellular kinase enzymes. FTC and phosphorylated metabolite give varying pharmacokinetic results [40]. The molecules mimic normal nucleos $(\mathrm{t})$ ides that are incorporated into DNA at the 3' terminus. However FTC and TNF lack the 3'-OH and their incorporation at the $3^{\prime}$ terminus of the DNA therefore terminates chain elongation by preventing incorporation of additional nucleotides [28, 41, 42]. TDF diphosphate and FTC 5'-triphosphate are weak inhibitors of $\alpha, \beta, \curlyvee$ cellular DNA polymerases and FTC 5'triphosphate weakly inhibits DNA polymerase $\varepsilon$ [28]. The combination of TNF and FTC has been the preferred NRTI regimen since 2003 since approval by FDA [30]. A single-tablet combination has been approved by the FDA and more recently by the Medicines Control Council (MCC) of South Africa for pre-exposure prophylaxis (PrEP) although it is not widely used clinically [43, 44]. The HIV-1 reverse transcriptase (RT) mutation K65R is a common multi-drug resistance mutation that confers resistance to NRTI including TNF and FTC $[11,42,45,46]$ and this mutation may be responsible for cross-resistance between different NRTI $[42,46]$. Cases of acute renal failure and Fanconi syndrome (FS) have been reported in patients treated with TNF [47] although clinically important renal toxicity is rare [48]. FTC and TNF undergo limited systemic metabolism [49].

\section{Tenofovir disoproxil fumarate (TDF)}

TDF is the acyclic nucleotide analogue of adenosine monophosphate approved for HIV treatment in 2004 [50]. Chemically, it is an [[2R]1-[6-aminopurin-9-yl]propan-2-yl]oxymethyl phosphonic acid, the solubility of which in water is $13.4 \mathrm{mg} / \mathrm{ml}$ at $25^{\circ} \mathrm{C}$ with a $\log P$ value of-1.6. TDF has an empirical formula of $\mathrm{C}_{19} \mathrm{H}_{30} \mathrm{~N}_{5} \mathrm{O}_{10} \mathrm{P}, \mathrm{C}_{4} \mathrm{H}_{4} \mathrm{O}_{4}$ and a relative molar mass of $635.5 \mathrm{~g} / \mathrm{mol}$. It occurs as a white to almost white crystalline powder [51-53]. TDF contains no less than 98.5 percent and not more than 101.0 percent of TDF $\left(\mathrm{C}_{19} \mathrm{H}_{30} \mathrm{~N}_{5} \mathrm{O}_{10} \mathrm{P}\right.$, $\mathrm{C}_{4} \mathrm{H}_{4} \mathrm{O}_{4}$ ), calculated with reference to an anhydrous reference material [52]. TDF refers to the solid/raw material whereas TNF refers to TDF in solution and tenofovir peaks in chromatograms. TDF is phosphorylated twice to the active diphosphate form. High fat meal increases the bioavailability of TDF and remains unaffected by normal meal [54]. As TDF is eliminated through the kidney and is not a substrate for CYP45, its dosage regimen needs to be modified in renal complications $[55,56]$. Gervasoni et al. showed that HIVinfected females with diminished body weight are in danger to be exposed to elevated TDF plasma trough concentrations, eventually bringing about a huge threat to produce long-term TDF complications [55].

\section{Non-nucleoside reverse transcriptase inhibitor [NNRTI]}

NNRTIs restrain the process of viral DNA synthesis by directly binding to the hydrophobic pocket of reverse transcriptase enzyme [31]. Unlike NRTIs, which must be phosphorylated to prevent HIV from infecting the cell, NNRTIs are active in the form administered. NNRTIs are classified as 1st generation and 2nd generation NNRTIs. $1^{\text {st }}$ generation NNRTIs include Nevirapine and Efavirenz [EFV] and $2^{\text {nd }}$ generation NNRTIs are Etravirine and Rilpivirine. HIV-2 is naturally resistant to NNRTIs.

\section{Efavirenz (EFV)}

EFV is a benzoxazin analogue approved by FDA in 1998 for the treatment of patients infected with HIV [57]. Chemically, it is an [4S]-6-chloro4-[2-cyclopropylethynyl]-4-[trifluoromethyl]-1H3,1benzoxazin-2-one and occurs as a white to slightly pink crystalline powder [58-60]. The empirical formula for $\mathrm{EFV}$ is $\mathrm{C}_{14} \mathrm{H}_{9} \mathrm{ClF}_{3} \mathrm{NO}_{2}$ and the relative molar mass is $315.7 \mathrm{~g} / \mathrm{mol}[58,60,61]$. EFV contains no less than 97.0 percent and not more than 103.0 percent of $\mathrm{C}_{14} \mathrm{H}_{9} \mathrm{ClF}_{3} \mathrm{NO}_{2}$ calculated with reference to the anhydrous reference material [58]. The solubility of which in water is $0.093 \mathrm{mg} / \mathrm{l}$ at $25^{\circ} \mathrm{C}$ with $\log \mathrm{P}$ value of 4.6. The dosing of EFV is once-daily due to its long half-life. EFV is usually preferred to treat HIV patients co-infected with tuberculosis [TB]. Both the diseases are life-threatening and treatment becomes very difficult due to drug-drug interactions 
between EFV and rifampicin [62,63]. Side effects of EFV are found to be associated with the EFV plasma concentration. Various side effects are associated with high and low plasma levels of EFV particularly in HIV-TB co-infected patients for which TDM studies become necessary. EFV levels are directly correlated with optimum therapeutic output and central nervous system side effects. Therefore, TDM of EFV in clinical practice is essential for optimum therapeutic output, especially in HIV-TB co-infected patients who are under treatment with the combination of EFV and rifampicin. On the other hand, EFV possesses high protein binding property [>99\%] and thus gets penetrated into male genital tract through blood. High penetration in male genital tract makes it an important candidate to study its concentration for prophylaxis use. HIV replication took place inside the cell, so ARV drugs have to enter the cells at an adequate concentration to restrain viral replication. Subsequently, studying intracellular drug concentration is a valuable tool to ascertain effective levels of ARVs in target cells mainly in virological failure regardless of efficient plasma level concentrations.

\section{Dissociation constant (pKa)}

EFV is a weak acid with a pKa of 10.2. It is therefore ionised at high $\mathrm{pH}$, at which the carbonate moiety undergoes deprotonation to form a negatively charged species. The trifluoromethyl and ethylene moieties are most likely responsible for the lowering of the pKa [64,
65]. The pKa of FTC and TNF are 2.65 and 3.75 respectively [35, 36, 66]. EFV is a weak acid whereas FTC and TNF are weak bases.

\section{Solubility}

EFV is practically insoluble in water $(9.0 \mu \mathrm{g} / \mathrm{ml})$ but is freely soluble in methanol $[14,31,38,39]$. FTC is freely soluble in methanol and water $(112 \mathrm{mg} / \mathrm{ml})$ and is practically insoluble in dichloromethane $\mathrm{R}$ [34-36, 58]. TNF has a solubility of $13.4 \mathrm{mg} / \mathrm{ml}$ in distilled water at $25^{\circ} \mathrm{C}$ [51].

\section{Biopharmaceutical classification system (BCS)}

The BCS provides a framework to classify molecules into categories based on their aqueous solubility and membrane permeability. Class 1 drugs have high solubility and high permeability, class 2 drugs low solubility and high permeability, class 3 drugs high solubility and low permeability and class 4 drugs low solubility and low permeability. EFV has low aqueous solubility and high intestinal permeability and is classified as a Class 2 molecule. FTC has high aqueous solubility and high intestinal permeability and is classified as a Class 1 molecule. TNF, the form of tenofovir that is aborbed, has high aqueous solubility and low intestinal permeability and is classified as a Class 3 molecule $[67,68]$

\section{Melting range}

EFV melts within the range of $139-141^{\circ} \mathrm{C}$ [69]. FTC melts within the range of $136-140{ }^{\circ} \mathrm{C}[70]$. TNF melts within the range of $276-280^{\circ} \mathrm{C}[71]$.<smiles>Nc1ncnc2c1ncn2C[C@H]1COP(O)(O)(O)CO1</smiles>

(A)

(B)

(C)

Fig. 1: Chemical structure of (A) Emtricitabine, (B) Tenofovir, (C) Efavirenz, reported analytical methods for TDF, FTC and EFV

\section{Spectrophotometric methods}

Many analytical methods involving spectroscopic analysis of the drug individually and as multicomponent samples have been reported. These methods include a simultaneous equation method, derivative spectrophotometric method, absorption ratio and a method based on $\mathrm{Q}$ analysis.

\section{Chromatographic method}

Liquid chromatographic analysis for the determination of TDF, FTC and EFV individually and in combination has been reported covering different phases of analytical research viz; profiling of impurities, stability indicating analytical methods, bioanalytical method development in different biological fluids to determine the concentration of TDF, FTC and EFV in human serum and to determine simultaneously in synthetic mixture or combination dosage form.

\section{Stability indicating method}

Stability indicating method is used to check drug stability under different conditions. Here, TDF, FTC and EFV are studied by RPHPLC and UPLC for stability studies.

Table 1: RP-HPLC/UPLC methods for determination of TDF, FTC and EFV

\begin{tabular}{|c|c|c|c|c|c|}
\hline S. No. & $\begin{array}{l}\text { Name of drug/formulation } \\
\text { /biological fluid }\end{array}$ & Column & Mobile phase composition & $\begin{array}{l}\text { Detection } \\
\text { (nm) }\end{array}$ & Ref. \\
\hline 1 & FTC-Tablet & $\begin{array}{l}\text { Peerless basic } \mathrm{C}_{18}(50 \mathrm{~mm} \times 4.6 \mathrm{~mm}, \\
3 \mu \mathrm{m})\end{array}$ & Buffer (pH 3.0): methanol-90:10\% (v/v) & $280 \mathrm{~nm}$ & 72 \\
\hline 2 & FTC-Nanoparticles & $\begin{array}{l}\text { Phenomenex } \mathrm{C}_{18}(250 \mathrm{~mm} \times 4.6 \mathrm{~mm} \text {, } \\
5 \mu \mathrm{m})\end{array}$ & $\begin{array}{l}40 \mathrm{mmol} \text { phosphate buffer ( } \mathrm{pH} 6.8) \text {, methanol } \\
\text { and } 2 \% \text { acetonitrile }(83: 15: 2, \mathrm{v} / \mathrm{v} / \mathrm{v})\end{array}$ & $280 \mathrm{~nm}$ & 73 \\
\hline 3 & FTC-Tablet & $\begin{array}{l}\text { Phenomenex } \mathrm{C}_{18}(250 \mathrm{~mm} \times 4.6 \mathrm{~mm} \text {, } \\
5 \mu \mathrm{m})\end{array}$ & $\begin{array}{l}10 \text { mmol phosphate buffer }(\mathrm{pH} 6.8) \text { methanol- } \\
2 \% \text { acetic acid (73: } 25: 2, \mathrm{v} / \mathrm{v} / \mathrm{v})\end{array}$ & $280 \mathrm{~nm}$ & 74 \\
\hline 4 & FTC-Capsule & Luna RP-18(2),250X4.6 mm, $5 \mu \mathrm{m}$ & Buffer: acetonitrile $(85: 15 \% \mathrm{v} / \mathrm{v})$ & $280 \mathrm{~nm}$ & 75 \\
\hline 5 & FTC-Capsule & $\begin{array}{l}\text { Phenomenex (Torrance, CA) С } 250 \times \\
4.6 \mathrm{~mm}\end{array}$ & $\begin{array}{l}\text { 0.03M Phosphate buffer }(\mathrm{pH} 4.86 \pm 0.02): \\
\text { acetonitrile: methanol }(40: 20: 40 \mathrm{v} / \mathrm{v} / \mathrm{v})\end{array}$ & $280 \mathrm{~nm}$ & 76 \\
\hline 6 & $\begin{array}{l}\text { FTC/TDF/Elvitegravir/Cobici } \\
\text { stat-Tablet }\end{array}$ & $\begin{array}{l}\text { Inertsil ODS } 3 V C_{18}(250 \mathrm{~mm} \times 4.6 \mathrm{~mm} \text {, } \\
5 \mu \mathrm{m}, 100 \AA)\end{array}$ & $\mathrm{A}=\mathrm{KH}_{2} \mathrm{PO}_{4}(0.02 \mathrm{M}) \mathrm{pH} 2.5, \mathrm{~B}=$ acetonitrile & $240 \mathrm{~nm}$ & 77 \\
\hline 7 & FTC/TDF-Tablet & Hypersil, 250 X $4.6 \mathrm{~mm}, 5 \mu$ & Buffer (pH 3.7): acetonitrile 60:40 (v/v) & - & 78 \\
\hline 8 & FTC/TDF/Rilpivirine-Tablet & Inertsil $\mathrm{C}_{18}(150 \times 4.6 \mathrm{~mm}, 5 \mu \mathrm{m})$ & $\begin{array}{l}\text { 0.1N Phosphate buffer(pH: 4): acetonitrile } \\
(40: 60 \mathrm{v} / \mathrm{v})\end{array}$ & $275 \mathrm{~nm}$ & 79 \\
\hline 9 & FTC/TDF-Tablet & Inspire $\mathrm{C}_{18}(150 \times 4.6 \mathrm{~mm}) 5.0 \mu \mathrm{m}$ & Buffer (pH 2.5): methanol (30:70 v/v) & $272 \mathrm{~nm}$ & 80 \\
\hline 10 & FTC/TDF-Tablet & Inspire $\mathrm{C}_{18}(4.6 \times 250 \mathrm{~mm}) 5 \mu \mathrm{m}$ & $\begin{array}{l}\text { Mixed buffer }\left(\mathrm{KH}_{2} \mathrm{PO}_{4} \text { and } \mathrm{K}_{2} \mathrm{HPO}_{4}\right) \mathrm{pH} 3: \mathrm{ACN} \\
(30: 70 \mathrm{v} / \mathrm{v})\end{array}$ & $273 \mathrm{~nm}$ & 81 \\
\hline 11 & FTC/TDF/Rilpivirine-Tablet & Kromasil $\mathrm{C}_{18}(250 \mathrm{~mm} \times 4.6 \mathrm{~mm}, 5 \mu)$ & $\begin{array}{l}0.01 \mathrm{~N} \text { Potassium dihydrogen phosphate and } \\
\text { acetonitrile } 65: 35(\mathrm{v} / \mathrm{v} /)\end{array}$ & $279 \mathrm{~nm}$ & 82 \\
\hline
\end{tabular}




\begin{tabular}{|c|c|c|c|c|c|}
\hline 12 & $\begin{array}{l}\text { FTC/TDF/Elvitegravir/Cobici } \\
\text { stat-Tablet }\end{array}$ & Atlantis $\mathrm{C}_{18}(100 \times 4.6 \mathrm{~mm}, 5 \mu \mathrm{m})$ & $\begin{array}{l}\text { Gradient mixture of } 0.1 \% \text { trifluoroacetic acid } \\
\text { and acetonitrile }\end{array}$ & $240 \mathrm{~nm}$ & 83 \\
\hline 13 & FTC/TDF-Plasma & Hypersil $\mathrm{C}_{18}(250 \mathrm{~mm} \times 4.0 \mathrm{~mm}, 5 \mu \mathrm{m})$ & $\begin{array}{l}\text { sodium dihydrogen orthophosphate buffer }(\mathrm{pH} \\
6.9) \text { and methanol }(96: 4)\end{array}$ & $\begin{array}{l}259 \\
265,280 \\
\mathrm{~nm}\end{array}$ & 84 \\
\hline 14 & FTC/TDF/Rilpivirine & $\begin{array}{l}\text { Thermo Hypersil ODS } C_{18}(150 \times 4.6 \\
m m, 5 \mu)\end{array}$ & Acetonitrile and Phosphate buffer pH 3 (60:40) & $260 \mathrm{~nm}$ & 85 \\
\hline 15 & FTC/TDF-Tablet & DIKMA $(50 \times 2.1 \mathrm{~mm}, 1.7 \mu)$ & Phosphate buffer (pH 5.6) and methanol 60:40 & $240 \mathrm{~nm}$ & 86 \\
\hline 16 & FTC/TDF-Tablet & $\begin{array}{l}\text { Hypersil TM BDS } \mathrm{C}_{18} 120 \mathrm{~A}(250 \times 4.60 \\
\mathrm{mm}, 5 \mu)\end{array}$ & $\begin{array}{l}\text { Methonal and phosphate buffer pH } 2.5 \text { (65:35 } \\
\% \mathrm{v} / \mathrm{v})\end{array}$ & $261 \mathrm{~nm}$ & 87 \\
\hline 17 & FTC/TDF/Bictegravir-Tablet & Zodiac $\mathrm{C}_{18} 150 \times 4.6 \mathrm{~mm}, 5 \mu$ & Buffer and Acetonitrie $(55: 45 \mathrm{v} / \mathrm{v})$ & $272 \mathrm{~nm}$ & 88 \\
\hline 18 & FTC/TDF-Tablet & $\begin{array}{l}\text { Phenomenex-Luna } \mathrm{C}_{18}(25 \mathrm{~cm} \times 4.60 \\
\mathrm{mm}, 5 \mu \mathrm{m})\end{array}$ & $\begin{array}{l}10 \mathrm{mmol} \text { phosphate buffer }(\mathrm{pH} 6.8): \\
\text { acetonitrile; } 40: 60(\mathrm{v} / \mathrm{v})\end{array}$ & $260 \mathrm{~nm}$ & 89 \\
\hline 19 & $\begin{array}{l}\text { FTC/TDF/Elvitegravir/Cobici } \\
\text { stat-Tablet }\end{array}$ & ODS $(250 \times 4.6 \mathrm{~mm}, 5 \mu \mathrm{m})$ & $\begin{array}{l}\mathrm{A}=\text { (potassium dihydrogen orthophosphate, } \mathrm{pH} \\
2.5 \text { ) } \\
\mathrm{B}=(\text { acetonitrile) } 55: 45 \% \mathrm{v} / \mathrm{v}\end{array}$ & $250 \mathrm{~nm}$ & 90 \\
\hline 20 & FTC/TDF-Tablet & $\begin{array}{l}\text { Phenomenax Luna } \mathrm{C}_{18}(150 \mathrm{~mm} \times 4.6 \\
\mathrm{mm}, 5 \mu \mathrm{m})\end{array}$ & Acetonitrile: methanol: water 30:50:20 (v/v) & $258 \mathrm{~nm}$ & 91 \\
\hline 21 & FTC/TDF-Tablet & $\mathrm{BEH} \mathrm{C}_{18}(100 \mathrm{~mm} \times 2.1,1.8 \mu \mathrm{m})$ & $\begin{array}{l}0.68 \% \text { potassium dihydrogen orthophosphate } \\
\text { buffer of } \mathrm{pH}=6 \text { and methanol } 45: 55 \mathrm{v} / \mathrm{v}\end{array}$ & $261 \mathrm{~nm}$ & 92 \\
\hline 22 & FTC/TDF-Tablet & Luna $\mathrm{C}_{18}(25 \mathrm{~cm} \times 4.60 \mathrm{~mm}, 5 \mu \mathrm{m})$ & $\begin{array}{l}\text { Acetonitrile: potassium dihydrogen phosphate } \\
\text { buffer }(\mathrm{pH} 3.0 \pm 0.05): \text { triethylamine } \\
70: 30: 0.5(\mathrm{v} / \mathrm{v})\end{array}$ & $260 \mathrm{~nm}$ & 93 \\
\hline 23 & FTC/TDF-Tablet & Promosil $\mathrm{C}_{18},(250 \mathrm{~mm}, 4.6 \mathrm{~mm}, 5 \mu \mathrm{m})$ & Methanol: Phosphate cushion 68:32 \% v/v. & $259 \mathrm{~nm}$ & 94 \\
\hline 24 & FTC/TDF-Tablet & $\begin{array}{l}\text { Inertsil ODS } C_{18}(250 \mathrm{~mm} \times 4.6 \mathrm{~mm}, 5 \\
\mu \mathrm{m})\end{array}$ & $\begin{array}{l}\text { 0.1\% triflouro acetic acid (TFA) buffer and } \\
\text { methanol 39:61 (v/v) }\end{array}$ & $261 \mathrm{~nm}$ & 95 \\
\hline 25 & $\begin{array}{l}\text { FTC/TDF/Cobicistat/Elvitegr } \\
\text { avir-Tablet }\end{array}$ & Kromasil $\mathrm{C}_{18}(250 \times 4.6 \mathrm{~mm}, 5 \mu \mathrm{m})$ & $\begin{array}{l}\text { Orthophosphoric acid buffer: acetonitrile } \\
(55: 45 \% \mathrm{v} / \mathrm{v})\end{array}$ & $240 \mathrm{~nm}$ & 96 \\
\hline 26 & FTC/TDF-Tablet & $\begin{array}{l}\text { Phenomenax Luna } \mathrm{C}_{18}(250 \mathrm{~mm} \times 4.6 \\
\mathrm{mm}, 5 \mu \mathrm{m})\end{array}$ & Methanol: phosphate buffer $\mathrm{pH}-3$ (70:30 v/v) & $258 \mathrm{~nm}$ & 97 \\
\hline 27 & $\begin{array}{l}\text { FTC/TDF/Dolutegravir- } \\
\text { Tablet }\end{array}$ & $\begin{array}{l}\text { Phenomenex kinetex Biphenyl } \\
250 \times 4.6 \mathrm{~mm}, 5 \mu \mathrm{m}\end{array}$ & $\begin{array}{l}\mathrm{A}=\text { ammonium acetate }(10 \mathrm{mmol}) \mathrm{pH} 3.0, \mathrm{~B}= \\
\text { Acetonitrile, ammonium acetate }(10 \mathrm{mmol}) \mathrm{pH} \\
3.0 \text { and methanol } 70: 15: 15 \% \mathrm{v} / \mathrm{v} / \mathrm{v}\end{array}$ & $260 \mathrm{~nm}$ & 98 \\
\hline 28 & $\begin{array}{l}\text { FTC/TDF/Cobicistat, } \\
\text { Elvitegravir-Tablet }\end{array}$ & $\begin{array}{l}\text { Kromasil } \mathrm{C}_{18}(250 \mathrm{~mm} \times 4.6 \mathrm{~mm} \times 5 \\
\mu \mathrm{m})\end{array}$ & $\begin{array}{l}0.01 \mathrm{~N} \mathrm{KH}_{2} \mathrm{PO}_{4}(\mathrm{pH} 2.5) \text { and acetonitrile } \\
(43: 57 \mathrm{v} / \mathrm{v})\end{array}$ & $254 \mathrm{~nm}$ & 99 \\
\hline 29 & $\begin{array}{l}\text { FTC/TDF/Cobicistat, } \\
\text { Elvitegravir-Tablet }\end{array}$ & $\begin{array}{l}\text { Hypersil BDS } \mathrm{C}_{18} 250 \times 4.6 \mathrm{~mm}, 5 \mu \text {, } \\
\text { 100A. }\end{array}$ & $\begin{array}{l}\text { 0.05M Phosphate buffer } \mathrm{pH} 3.0 \text { and } \\
\text { acetonitrile } 95: 5\end{array}$ & $240 \mathrm{~nm}$ & 100 \\
\hline 30 & FTC/TDF/Rilpivirine-Tablet & Agilent $\mathrm{C}_{18}(250 \times 4.6 \mathrm{~mm}, 5 \mu \mathrm{m})$ & $0.1 \%$ Formic acid: acetonitrile $(65: 35 \%, \mathrm{v} / \mathrm{v})$ & $250 \mathrm{~nm}$ & 101 \\
\hline 31 & TDF-Tablet & $\begin{array}{l}\text { Phenomenex Luna } \mathrm{C}_{18}(250 \times 4.6 \mathrm{~mm} \times \\
5 \mu \mathrm{m})\end{array}$ & $\begin{array}{l}\text { Orthophosphoric acid (pH 3.0): acetonitrile: } \\
\text { aethanol }(40: 50: 10 \% \mathrm{v} / \mathrm{v})\end{array}$ & $254 \mathrm{~nm}$ & 102 \\
\hline 32 & TDF/lamivudine-Tablet & $\begin{array}{l}\text { HypersilTM BDS C } 18 \text { 120A }(250 \times 4.60 \\
\mathrm{mm}, 5 \mu)\end{array}$ & $\begin{array}{l}\text { Acetonitrile and phosphate buffer pH } 3.5 \\
(80: 20 \% \mathrm{v} / \mathrm{v})\end{array}$ & $260 \mathrm{~nm}$ & 103 \\
\hline 33 & TDF/lamivudine-Tablet & $\begin{array}{l}\text { Inertsil } C_{18} \text { column }(15 \mathrm{~cm} \mathrm{x} 4.6 \mathrm{~mm}, 5 \\
\mu \mathrm{m})\end{array}$ & $\begin{array}{l}6.5 \mathrm{mmol} \text { Phosphate buffer } \mathrm{pH} 2.5 \text { and } \\
\text { acetonitrile }(50: 50 \mathrm{v} / \mathrm{v})\end{array}$ & $260 \mathrm{~nm}$ & 104 \\
\hline 34 & TDF-Tablet & $\begin{array}{l}\text { Acquity UPLC BEH C } 18(1.7 \mu \mathrm{m}, 1 \mathrm{~mm} \\
\text { X } 50 \mathrm{~mm})\end{array}$ & $0.1 \%$ Formic acid and acetonitrile & - & 105 \\
\hline 35 & TDF/lamivudine & $\begin{array}{l}\text { Waters X-terra } \mathrm{RPC}_{18}(150 \times 4.6 \mathrm{~mm} \text {, } \\
3.5 \mu \mathrm{m})\end{array}$ & $\begin{array}{l}A=(\text { ammonium acetate buffer, } \mathrm{pH} 5.0 \pm 0.05) \\
\text { and } \mathrm{B}=(\text { methanol and ammonium acetate } \\
\text { buffer } 20: 80)\end{array}$ & $260 \mathrm{~nm}$ & 106 \\
\hline 36 & TDF/lamivudine-Tablet & $\begin{array}{l}\mathrm{C}_{18}(\text { Inertsil ODS 3V, } 250 \mathrm{~mm} \times 4.6 \\
\mathrm{mm} ; 5 \mu)\end{array}$ & $\begin{array}{l}\text { Phosphate buffer (pH3.5) and acetonitrile } \\
55: 45 \mathrm{v} / \mathrm{v}\end{array}$ & $264 \mathrm{~nm}$ & 107 \\
\hline 37 & TDF-Tablet & $\begin{array}{l}\text { HiQ Sil } \mathrm{C}_{18} \mathrm{HS}(250 \mathrm{~mm} \times 4.6 \mathrm{~mm}, 5.0 \\
\mu \mathrm{m})\end{array}$ & Methanol: water $(60: 40, \mathrm{v} / \mathrm{v})$ & $260 \mathrm{~nm}$ & 108 \\
\hline 38 & EFV-Tablet & Welchrom $\mathrm{C}_{18}(4.6 \times 250 \mathrm{~mm}, 5 \mu \mathrm{m})$ & $\begin{array}{l}10 \text { mmol Phosphate buffer }(\mathrm{pH} 3.0): \\
\text { acetonitrile }(50: 50 \mathrm{v} / \mathrm{v})\end{array}$ & $246 \mathrm{~nm}$ & 109 \\
\hline 39 & EFV-Plasma & $\begin{array}{l}\text { Waters X-Terra Shield, } C_{18} 50 \times 4.6 \\
\mathrm{~mm}, 3.5 \mu \mathrm{m}\end{array}$ & Phosphate buffer pH 3.5 and acetonitrile & $260 \mathrm{~nm}$ & 110 \\
\hline 40 & $\begin{array}{l}\text { EFV/Lamivudine/Stavudine- } \\
\text { Tablet }\end{array}$ & Inertsil ODS $\mathrm{C}_{18}(4.6 \times 250 \mathrm{~mm}, 5.0 \mu \mathrm{m})$ & Phosphate Buffer (pH 4): methanol 30:70 v/v & $254 \mathrm{~nm}$ & 111 \\
\hline 41 & EFV-Tablet & $\mathrm{C}_{18} 250 \times 4 \mathrm{~mm}(10 \mu \mathrm{m})$ & ACN: water: $85 \% \mathrm{H}_{3} \mathrm{PO}_{4}(70: 30: 0.1)$ & $252 \mathrm{~nm}$ & 112 \\
\hline 42 & EFV-Tablet & $\begin{array}{l}\text { Develosil ODS HG-5 RP } 150 \mathrm{~mm} \times 4.6 \\
\mathrm{~mm} 5 \mu \mathrm{m}\end{array}$ & $\begin{array}{l}\text { Phosphate buffer ( } \mathrm{pH} 3.1) \text { and acetronitrile } \\
\text { 40:60. }\end{array}$ & $249 \mathrm{~nm}$ & 113 \\
\hline 43 & EFV-Tablet & Waters XBridge $(4.6 \times 250 \mathrm{~mm}, 5 \mu \mathrm{m})$ & $\begin{array}{l}\text { Ammonium format buffer ( } \mathrm{pH} 5) \text { : ACN (28:72 } \\
\mathrm{v} / \mathrm{v})\end{array}$ & $247 \mathrm{~nm}$ & 114 \\
\hline 44 & EFV/Lamivudine-Tablet & $\begin{array}{l}\text { Acquity UPLC BEH Shield RP18 }(50 \times 3 \\
\mathrm{mm}, 1.7 \mu \mathrm{m})\end{array}$ & $\begin{array}{l}10 \% \text { acetonitrile in methanol and } 10 \mathrm{mmol} \\
\text { phosphate buffer }(\mathrm{pH} 4.0)\end{array}$ & $254 \mathrm{~nm}$ & 115 \\
\hline 45 & EFV/TDF/lamivudine-Tablet & $\begin{array}{l}\text { Kromasil } \mathrm{C}_{18} \text { analytical column }(150 \times \\
4.6 \mathrm{~mm}, 5 \mu \mathrm{m})\end{array}$ & $\begin{array}{l}10 \text { mmol phosphate buffer ( } \mathrm{pH} 5.0) \text { and } \\
\text { methanol (30:70) }\end{array}$ & $254 \mathrm{~nm}$ & 116 \\
\hline 46 & EFV/TDF/lamivudine-Plasma & Luna $\mathrm{C}_{18}(250 \times 4.6 \mathrm{~mm}, 5 \mu)$ & $\begin{array}{l}\text { A: Water with } 0.1 \% \text { (THF); B: acetonitrile with } \\
0.1 \% \text { THF }\end{array}$ & $254 \mathrm{~nm}$ & 117 \\
\hline 47 & EFV/TDF/lamivudine-Tablet & SHISEIDO $\mathrm{C}_{18}(250 \times 4.6 \mathrm{~mm}, 5 \mu)$ & $\begin{array}{l}\text { Acetonitrile: } 50 \mathrm{mmol} \text { phosphate buffer (pH } \\
5.0 \text { ) }\end{array}$ & $256 \mathrm{~nm}$ & 118 \\
\hline 48 & FTC/TDF/EFV-Tablet & $\begin{array}{l}\text { Zorbax SB-Phenyl, ( } 250 \mathrm{~mm} \mathrm{X} 4.6 \\
\mathrm{~mm}), 5 \mu \mathrm{m}\end{array}$ & $\begin{array}{l}\text { A: Buffer pH } 3.7 \mathrm{~B} \text { : methanol, acetonitrile and } \\
\text { tetrahydrofuran (50: } 50: 2 \mathrm{v} / \mathrm{v} / \mathrm{v})\end{array}$ & $265 \mathrm{~nm}$ & 119 \\
\hline 49 & FTC/TDF/EFV-Tablet & Inertsil ODS $3 \mathrm{~V}(250 \times 4.6 \mathrm{~mm}, 5 \mu)$ & $\begin{array}{l}\text { A: } 0.02 \mathrm{M} \text { Sodium dihydrogen orthophosphate } \\
\text { monohydrate B: methanol and water }(85: 15)\end{array}$ & $265 \mathrm{~nm}$ & 120 \\
\hline 50 & FTC/TDF/EFV-Plasma & $\begin{array}{l}\text { Chromolith Performance RP-18e }(100 \\
\times 4.6 \mathrm{~mm})\end{array}$ & A: $(0.1 \%$ formic acid $), B$ : acetonitrile & - & 121 \\
\hline
\end{tabular}




\begin{tabular}{|c|c|c|c|c|c|}
\hline 51 & FTC/TDF/EFV & $\begin{array}{l}\text { Acquity UPLC BEH m) c } \mu \text { Phenyl } \\
(2.1 \times 100 \mathrm{~mm}, 1.7\end{array}$ & $\begin{array}{l}\text { A: (Buffer, } 1.0 \mathrm{ml} \text { of TEA in } 1000 \mathrm{ml} \text { of water } \\
\text { pH } 4.0 \pm 0.05) \text { and B: (buffer: acetonitrile } \\
(20: 80, \mathrm{v} / \mathrm{v})\end{array}$ & $265 \mathrm{~nm}$ & 122 \\
\hline 52 & FTC/TDF/EFV-Tablet & $\begin{array}{l}\text { Phenomenex } \mathrm{C}_{8}(250 \mathrm{~mm} \times 4.6 \mathrm{~mm}, 5 \\
\mu \mathrm{m})\end{array}$ & $\begin{array}{l}\text { Buffer pH: } 7.0 \text { acetonitrile and methanol } \\
(40: 40: 20 \mathrm{v} / \mathrm{v})\end{array}$ & $262 \mathrm{~nm}$ & 123 \\
\hline 53 & FTC/TDF/EFV-Tablet & Kromosil $C_{18} 100 \times 4.6,3.5 \mu$ & $\begin{array}{l}0.1 \mathrm{M} \text { ortho phosphoric acid buffer and } \\
\text { acetonitrile in } 60: 40 \mathrm{v} / \mathrm{v}\end{array}$ & $265 \mathrm{~nm}$ & 124 \\
\hline 54 & FTC/TDF/EFV-Tablet & $\begin{array}{l}\text { XTerra symmetry } C_{18}(4.6 \times 150 \mathrm{~mm} \text {, } \\
3.5 \mu \mathrm{m})\end{array}$ & Di hydrogen sulphate: methanol & $270 \mathrm{~nm}$ & 125 \\
\hline 55 & FTC/TDF/EFV-Tablet & $\begin{array}{l}\text { Zorbax } C_{8} \text { column }(150 \mathrm{~mm} \times 4.6 \mathrm{~mm} \text {, } \\
5 \mu \mathrm{m})\end{array}$ & Buffer pH $2.4 \pm 0.02$ and acetonitrile $70: 30 \mathrm{v} / \mathrm{v}$ & $252 \mathrm{~nm}$ & 126 \\
\hline 56 & FTC/TDF/EFV-Tablet & $\begin{array}{l}\text { Acquity } 50 \mathrm{~mm} \times \mathrm{m} 2.1 \mu \mathrm{UPLC} \text { BEH } \\
\text { Phenyl } 1.7\end{array}$ & $\begin{array}{l}\text { A: } 0.2 \% \text { TEA in water } \mathrm{pH} 6.5 \pm 0.05 \text {, B: methanol } \\
\text { and acetonitrile } 50: 50(\mathrm{v} / \mathrm{v})\end{array}$ & $260 \mathrm{~nm}$ & 127 \\
\hline 57 & FTC/TDF/EFV-Tablet & $\begin{array}{l}\text { Hypersil BDS } C_{18} \text { column }(25 \mathrm{~cm} \times 4.6 \\
\mathrm{mm} 5 \mu \mathrm{m})\end{array}$ & Acetonitrile, phosphate buffer and water & $280 \mathrm{~nm}$ & 128 \\
\hline 58 & FTC/TDF/EFV-Tablet & Thermosil $\mathrm{C}_{18}(100 * 4.6 \mathrm{~mm}, 5 \mu)$ & $\begin{array}{l}\text { Methanol and Triethylamine (pH 7) (70:30 } \\
\text { V/V }\end{array}$ & $260 \mathrm{~nm}$ & 129 \\
\hline 59 & FTC/TDF/EFV-Tablet & $\begin{array}{l}\text { Inertsil ODS } 3 \mathrm{~V} \mathrm{C}_{18}(150 \mathrm{~mm} \times 4.6 \\
\mathrm{mm} 5 \mu \mathrm{m})\end{array}$ & $\begin{array}{l}\text { Phosphate buffer (pH 3.5): acetonitrile (70: } 30 \\
\text { v/v) }\end{array}$ & $256 \mathrm{~nm}$ & 130 \\
\hline 60 & FTC/TDF/EFV-Tablet & Zorbax SB CN, $(250 \times 4.6 \mathrm{~mm}, 5 \mu \mathrm{m})$ & A: methanol, B: buffer at pH 4.5 & $260 \mathrm{~nm}$ & 131 \\
\hline 61 & FTC/TDF/EFV-Tablet & $\mathrm{HSS} \mathrm{C}_{18}(100 \times 3 \mathrm{~mm}, 1.7 \mu)$ & $\begin{array}{l}0.01 \mathrm{~N} \text { Phosphate buffer }(\mathrm{pH} 4.5) \text { and } \\
\text { acetonitrile }(40: 60, \mathrm{v} / \mathrm{v})\end{array}$ & $265 \mathrm{~nm}$ & 132 \\
\hline 62 & FTC/TDF/EFV-Tablet & Inerstil ODS $\mathrm{C}_{18} 250 \times 4.6 \mathrm{~mm}, 5 \mu \mathrm{m}$ & $\begin{array}{l}\text { A: Buffer }(0.05 \% \text { Trifluro acetic acid in water }) \\
\text { B: methanol }\end{array}$ & $262 \mathrm{~nm}$ & 133 \\
\hline 63 & FTC/TDF-Tablet & $\mathrm{C}_{18}(250 \times 4.6 \mathrm{~mm})$ & Methanol: distill water 60:40 v/v (pH-3) & $260 \mathrm{~nm}$ & 134 \\
\hline 64 & FTC/TDF/Rilpivirine-Tablet & $\begin{array}{l}\text { Inertsil ODS } 3 V C_{18}(250 \mathrm{~mm} \times 4.6 \mathrm{~mm} \text {, } \\
5 \mu \mathrm{m}) \text {, }\end{array}$ & $\begin{array}{l}0.01 \mathrm{M} \text { phosphate buffer }(\mathrm{pH} 4) \text { and } \\
\text { acetonitrile }(30: 70 \mathrm{v} / \mathrm{v})\end{array}$ & $265 \mathrm{~nm}$ & 135 \\
\hline 65 & FTC/TDF-Tablet & Hi Q $\mathrm{C}_{18} \mathrm{~W}(150 \mathrm{~mm}: 4.6 \mathrm{~mm}, 5 \mu)$ & Buffer, methanol and acetonitrile (40: 50: 10) & $\begin{array}{l}265,278 \\
\mathrm{~nm}\end{array}$ & 136 \\
\hline 66 & FTC/TDF/Rilpivirine-Tablet & Acquity $\mathrm{BEH}_{18}(50 \times 2.1 \mathrm{~mm}, 1.7 \mu \mathrm{m})$ & $\begin{array}{l}\text { Acetonitrile and phosphate buffer }(\mathrm{pH} \\
\text { 3) } 55: 45(\mathrm{v} / \mathrm{v}) \text {. }\end{array}$ & $261 \mathrm{~nm}$ & 137 \\
\hline 67 & $\begin{array}{l}\text { FTC/TDF/Lamivudine- } \\
\text { Plasma }\end{array}$ & ACE 5 CN $(150 \mathrm{~mm} \times 4.6 \mathrm{~mm}, 5 \mu \mathrm{m})$ & $\begin{array}{l}0.5 \% \text { Formic acid in water and acetonitrile } \\
(55: 45, \mathrm{v} / \mathrm{v})\end{array}$ & - & 138 \\
\hline 68 & FTC/TDF-Tablet & Premsil $\mathrm{C}_{18}(250 \mathrm{~mm} \times 4.6 \mathrm{~mm}, 5 \mu \mathrm{m})$ & Methanol: water $(70: 30 \mathrm{v} / \mathrm{v}) \mathrm{pH} 3$ & $273 \mathrm{~nm}$ & 139 \\
\hline 69 & FTC/TDF-Tablet & Zorbax SB-C $85 \mu \mathrm{m}, 4.6 \times 250 \mathrm{~mm}$ & $\begin{array}{l}50 \text { mmol Phosphate buffer }(\mathrm{pH} 6.0)- \\
\text { acetonitrile }(50: 50, \mathrm{v} / \mathrm{v})\end{array}$ & $\begin{array}{l}260,280 \\
\mathrm{~nm}\end{array}$ & 140 \\
\hline 70 & FTC & $\mathrm{RP}_{18}(25 \mathrm{~cm} \times 4.6 \mathrm{~mm}), 5 \mu \mathrm{m}$ & ACN, phosphate buffer ( $\mathrm{pH} 4.4)$, and water & $280 \mathrm{~nm}$ & 141 \\
\hline 71 & FTC/TDF-Plasma & Synergi Polar-RP, $2.0 \mathrm{~mm} \times 150 \mathrm{~mm}$, & $3 \%$ Acetonitrile $/ 1 \%$ acetic acid, aq.) & - & 142 \\
\hline 72 & FTC/TDF-Plasma & $\begin{array}{l}\text { Chromolith Speed Rod RPC } 18(50 \\
\mathrm{mm} \times 4.6 \mathrm{~mm})\end{array}$ & $\begin{array}{l}\text { Methanol,acetonitrile and ammonium acetate } \\
\text { (pH 3.0, } 40 \mathrm{mmol})(20: 80, \mathrm{v} / \mathrm{v})\end{array}$ & - & 143 \\
\hline 73 & FTC/TDF-seminal plasma & Atlantis T3 $\mathrm{C}_{18}(2.1 \times 100 \mathrm{~mm}, 3 \mu \mathrm{m})$ & $\begin{array}{l}\text { A: Deionized water with } 0.05 \% \text { formic acid, B: } \\
\text { methanol with } 0.05 \% \text { formic acid }\end{array}$ & - & 144 \\
\hline 74 & FTC/TDF-Tablet & Agilent TC- $\mathrm{C}_{18} 5 \mathrm{~mm}, 4.6^{\prime} 250 \mathrm{~mm}$ & $\begin{array}{l}\text { Methanol and phosphate buffer }(30: 70 \mathrm{v} / \mathrm{v}, \mathrm{pH} \\
\text { 4) }\end{array}$ & $261 \mathrm{~nm}$ & 145 \\
\hline 75 & FTC/TDF-Tablet & Inertsil ODS $\mathrm{C}_{18},(4.6 \times 250 \mathrm{~mm}, 5 \mu \mathrm{m})$ & Buffer: Acetonitrile (80:20) & $259 \mathrm{~nm}$ & 146 \\
\hline 76 & FTC/TDF/EFV-Tablet & Hypersil BDS $\mathrm{C}_{18}, 250 \times 4.6 \mathrm{~mm}, 5 \mu \mathrm{m}$ & $\begin{array}{l}\text { Acetonitrile and } 0.03 \mathrm{M} \mathrm{KH}_{2} \mathrm{PO}_{4}(\mathrm{pH} 3.2) 60: 40 \\
\mathrm{v} / \mathrm{v}\end{array}$ & $260 \mathrm{~nm}$ & 147 \\
\hline
\end{tabular}

Table 2: Spectrophotometric methods used for determination of TDF, FTC and EFV alone and in combined dosage form

\begin{tabular}{|c|c|c|c|c|c|}
\hline S. No. & Name of drug & Sample matrix & Method & Detection (nm) & Ref. \\
\hline 1 & EFV & Tablet & Zero order & 247 & 148 \\
\hline 2 & TDF & Tablet & Zero order & 260 & 149 \\
\hline 3 & EFV/TDF/Lamivudine & Tablet & Simultaneous equation & $247,260,272 \mathrm{~nm}$ & 150 \\
\hline 4 & FTC & Tablet & Zero order and area under curve & $280 \mathrm{~nm}, 272-286 \mathrm{~nm}$ & 151 \\
\hline 5 & TDF & Tablet & Zero order & $260 \mathrm{~nm}$ & 152 \\
\hline 6 & EFV/TDF/Lamivudine & Tablet & Zero order & $247,260,272 \mathrm{~nm}$ & 153 \\
\hline 7 & FTC/TDF & Bulk & $\begin{array}{l}\text { Simultaneous equation, Q- } \\
\text { absorbance ratio method }\end{array}$ & (1) $280 \mathrm{~nm}, 260 \mathrm{~nm}$ (2) $251,237 \mathrm{~nm}$ & 154 \\
\hline 8 & FTC/TDF & Tablet & Simultaneous equation eethod & $282,261 \mathrm{~nm}$ & 155 \\
\hline 9 & FTC/TDF & Tablet & Simultaneous Equation Method & $281,210 \mathrm{~nm}$ & 156 \\
\hline 10 & FTC/TDF/EFV & Tablet & Simultaneous equation method & $260,241,240 \mathrm{~nm}$ & 157 \\
\hline 11 & FTC/TDF/Cobicistat/Elvitegravir & Tablet & Simultaneous equation method & $283,259,240,258 \mathrm{~nm}$ & 158 \\
\hline 12 & FTC & Tablet & Zero order, first order derivative & 241.1 and $232.7 \mathrm{~nm}$ & 159 \\
\hline 13 & FTC/TDF & Tablet & $\begin{array}{l}\text { Least square, first order, area under } \\
\text { curve }\end{array}$ & $\begin{array}{l}281,260.5 \mathrm{~nm} ; 234.5,281 \mathrm{~nm} ; 278- \\
283 \mathrm{~nm}, 258-262 \mathrm{~nm}\end{array}$ & 160 \\
\hline 14 & TDF & Tablet & Zero order, first order & $260 \mathrm{~nm}, 273 \mathrm{~nm}$ & 161 \\
\hline 15 & EFV/TDF/Lamivudine & Tablet & $\begin{array}{l}\text { Simultaneous equation method, } \\
\text { multicomponent analysis and } \\
\text { derivative spectroscopy method }\end{array}$ & 247,259 and $272 \mathrm{~nm}$ & 162 \\
\hline 16 & EFV/TDF/Rilpivirine & Tablet & Simultaneous equation method & $240.8,257.6$, and $305.6 \mathrm{~nm}$ & 163 \\
\hline 17 & $\mathrm{EFV} / \mathrm{TDF}$ & Tablet & $\begin{array}{l}\text { Simultaneous equation and } \\
\text { Absorbance ratio method }\end{array}$ & $250,274 \mathrm{~nm} ; 255,274 \mathrm{~nm}$ & 164 \\
\hline 18 & $\mathrm{EFV} / \mathrm{TDF}$ & Tablet & $\begin{array}{l}\text { Ratio derivative spectra, first-order, } \\
\text { absorption corrected method }\end{array}$ & $\begin{array}{l}271.07 \text { and } 302.17 \mathrm{~nm} ; 224.38 \text { and } \\
306.88 \mathrm{~nm}\end{array}$ & 165 \\
\hline 19 & $\mathrm{EFV} / \mathrm{TDF}$ & Tablet & Zero order & $298 \mathrm{~nm}$ & 166 \\
\hline 20 & TDF/Lamivudine & Tablet & Simultaneous equation method & 271 and $261 \mathrm{~nm}$ & 167 \\
\hline
\end{tabular}


Table 3: HPTLC methods for determination of TDF, FTC and EFV

\begin{tabular}{|c|c|c|c|c|c|c|}
\hline S. No. & Name of drug & Formulation & $\begin{array}{l}\text { Stationary phase } \\
\text { plates }\end{array}$ & Mobile phase composition & $\mathbf{R f}$ & Ref. \\
\hline 1 & EFV/Lamivudine & Tablet & $\begin{array}{l}\text { silica gel 60G } \\
\text { F254 }\end{array}$ & $\begin{array}{l}\text { Ethyl acetate: methanol: } \\
\text { formic acid } 7.0: 2.5: 0.5(\mathrm{v} / \mathrm{v})\end{array}$ & $\begin{array}{l}\text { Lamivudine }=0.57 \pm 0.02 \\
\mathrm{EFV}=0.72 \pm 0.01\end{array}$ & 168 \\
\hline 2 & FTC/TDF/EFV & Tablet & silica gel 60F 254 & $\begin{array}{l}\text { Chloroform: methanol } \\
(90: 10)\end{array}$ & $\begin{array}{l}\mathrm{FTC}=0.15, \mathrm{TDF}=0.34, \mathrm{EFV} \\
=0.55\end{array}$ & 169 \\
\hline 3 & FTC/TDF/Rilpivirin & Tablet & silica gel 60 F254 & $\begin{array}{l}\text { Methanol: toluene: } \\
\text { ethylacetate: ammonia } \\
(1.5: 5.5: 1.5: 0.1 \mathrm{v} / \mathrm{v} / \mathrm{v} / \mathrm{v})\end{array}$ & $\begin{array}{l}\text { Rilpivirin }=0.59, \mathrm{FTC}= \\
=0.29, \mathrm{TDF}=0.41\end{array}$ & 170 \\
\hline 4 & TDF/Lamivudine & Tablet & $\begin{array}{l}\text { silica gel } 60 \mathrm{~F} 254 \text {, } \\
(20 \times 10 \mathrm{~cm})\end{array}$ & $\begin{array}{l}\text { Chloroform: methanol: } \\
\text { toluene }(8: 2: 2, \mathrm{v} / \mathrm{v} / \mathrm{v})\end{array}$ & $\begin{array}{l}\text { TDF }=0.51, \text { Lamivudine }= \\
0.27\end{array}$ & 171 \\
\hline 5 & EFV & Tablet & silica gel 60 F 254 & $\begin{array}{l}\text { Toluene: ethyl acetate: formic } \\
\text { acid }(10: 3: 1 \mathrm{v} / \mathrm{v})\end{array}$ & $0.41 \pm 0.01$ & 172 \\
\hline 6 & EFV & Plasma & silica gel 60F254 & $\begin{array}{l}\text { Dichloromethane: methanol } \\
(5: 0.3 \mathrm{v} / \mathrm{v})\end{array}$ & $0.69 \pm 0.01$ & 173 \\
\hline 7 & TDF & Tablet & $\begin{array}{l}\text { silica gel GF } \\
\text { aluminum }\end{array}$ & $\begin{array}{l}\text { Ethyl acetate: methanol: } \\
\text { formic acid( } 7: 2.5: 0.5 \% \mathrm{v} / \mathrm{v})\end{array}$ & 0.78 & 174 \\
\hline 8 & FTC/TDF/Rilpivirin & Tablet & silica gel $60 \mathrm{~F}_{254}$ & $\begin{array}{l}\text { Chloroform: ethyl acetate: } \\
\text { methanol: glacial acetic acid } \\
(5: 2: 1: 0.1 \mathrm{v} / \mathrm{v} / \mathrm{v} / \mathrm{v})\end{array}$ & $\begin{array}{l}\mathrm{FTC}=0.28, \mathrm{TDF}=0.52, \\
\text { Rilpivirin }=0.70\end{array}$ & 175 \\
\hline
\end{tabular}

\section{CONCLUSION}

The present review discussed about different analytical approach employed for the assessment of TDF, FTC and EFV. Profuse examinations have been accomplished including, Bio-analytical, HPLC, UPLC, HPTLC, UV/Vis-Spectroscopy, LC-MS, LC-ESI-MS etc. for evaluation of TDF, FTC and EFV in bulk and in its combination with other drugs from pharmaceutical formulations and also biological fluids. Liquid chromatography with UV detection has been found to be most studied for estimation of TDF, FTC and EFV in bulk as well as pharmaceutical dosage forms, while hyphenated LS-MS, LSMS/MS methods reported for determination of TDF, FTC and EFV and its metabolite in plasma and other biological fluids. Few chromatography approaches like HPTLC and Stability-indicating HPLC, UPLC and HPTLC are also reported. Few simple UV-Spectrophometric methods may be used for routine analysis of TDF, FTC and EFV alone and in combination with other drugs. These compiled data may of use for research for further studies in analysis of TDF, FTC and EFV.

\section{FUNDING}

Nil

\section{AUTHORS CONTRIBUTIONS}

All the authors have contributed equally.

\section{CONFLICT OF INTERESTS}

\section{Declared none}

\section{REFERENCES}

1. Parr MK, Schmidt AH. Life cycle management of analytical methods. J Pharm Biomed Anal 2018;147:506-17.

2. Gaudin K, Ferey L. Quality by design: a tool for separation method development in pharmaceutical laboratories. LC-GC 2016;29:16-25.

3. Maggio RM, Vignaduzzo SE, Kaufman TS. Practical and regulatory considerations for stability-indicating methods for the assay of bulk drugs and drug formulations. TrAC, Trends Anal Chem 2013;49:57-70.

4. Blessy M, Patel RD, Prajapati PN, Agrawal YK. Development of forced degradation and stability indicating studies of drugs-a review. J Pharm Anal 2014;4:159-65.

5. Singh S, Junwal M, Modhe G, Tiwari $\mathrm{H}$, Kurmi M, Parashar N, et al. Forced degradation studies to assess the stability of drugs and products. TrAC, Trends Anal Chem 2013;49:71-88.

6. ICH Harmonised Tripartite Guideline: Stability Testing of New Drug Substances and Products Q1A (R2), current Step 4 version; International Conference on Harmonisation: Geneva; 2003.

7. Global report: UNAIDS report on the global AIDS epidemic; 2012.
8. Zagury D, Bernard J, Leibowitch J, Safai B, Groopman JE, Feldman M, et al. HTLV-III in cells cultured from semen of two patients with AIDS. Science 1984;226:449-51.

9. Vogt MW, Witt DJ, Craven DE, Byington R, Crawford DF, Schooley RT, et al. Isolation of HTLV-III/lAV from cervical secretions of women at risk for AIDS. Lancet 1986;1:525-7.

10. Friedland GH, Klein RS. Transmission of the human immunodeficiency virus. N Engl J Med 1987;317:1125-35.

11. Chermann JC. Sexual and mother-to-child transmission of the human immunodeficiency virus type 1: a review. Am J Reprod Immunol 1998;40:183-6.

12. Mocroft A, Vella S, Benfield TL, Chiesi A, Miller V, Gargalianos P, et al. Changing patterns of mortality across Europe in patients infected with HIV-1. Euro SIDA Study Group. Lancet 1998;45:1093-9.

13. Palella FJ, Delaney KM, Moorman AC, Loveless MO, Fuhrer J, Satten GA, et al. Declining morbidity and mortality among patients with advanced human immunodeficiency virus infection. HIV outpatient study investigators. N Engl J Med 1998;338:853-60.

14. Vittinghoff E, Scheer S, O'Malley P, Colfax G, Holmberg SD, Buchbinder SP. Combination antiretroviral therapy and recent declines in AIDS incidence and mortality. J Infect Dis 1999;179:717-20.

15. Grant RM, Lama JR, Anderson PL, McMahan V, Liu AY, Vargas L, et al. Preexposure chemoprophylaxis for HIV prevention in men who have sex with men. N Engl J Med 2010;363:2587-99.

16. Cohen MS, Chen YQ, McCauley M, Gamble T, Hosseinipour MC, Kumarasamy N, et al. Prevention of HIV-1 infection with early antiretroviral therapy. N Engl J Med 2011;365:493-505.

17. Centers for Disease Control and Prevention (CDC). Interim guidance: preexposure prophylaxis for the prevention of HIV infection in men who have sex with men. MMWR Morb Mortal Wkly Rep 2011;60:65-8.

18. Baeten JM, Donnell D, Ndase P, Mugo NR, Campbell JD, Wangisi J, et al. Antiretroviral prophylaxis for HIV prevention in heterosexual men and women. N Engl J Med 2012;367:399-410.

19. Food, Drug Administration, FDA-approved ARV drugs; 2020.

20. Volberding PA, Deeks SG. Antiretroviral therapy and management of HIV infections. Lancet 2010;376:49-62.

21. Capetti A, Astuti N, Cossu MV, Rizzardini G, Carenzi L. The role of therapeutic drug monitoring and pharmacogenetic testing in the management of HIV infection: a review. J Aids Clin Res 2015;6:11-9.

22. Panel on Antiretroviral Guidelines for Adults and Adolescents. Guidelines for the use of antiretroviral agents in HIV-1-infected adults and adolescents. Department of Health and Human Services; 2015

23. Burcin B, Bengi U, Ozkan SA. A review of electroanalytical techniques for determination of anti-HIV drugs. Int J Electrochem 2011. https://doi.org/10.4061/2011/343947 
24. Echenique IA, Rich JD. EFV/FTC/TDF-associated hepatotoxicity: a case report and review. AIDS Patient Care STDs 2013;27:493-7.

25. Aspen Pharmacare, "TribussTM,"; 2011.

26. Zamisa T. HIV patients revel in the one pill a day age; 2014.

27. European Medicines Agency, Summary of product characteristics: Viread $245 \mathrm{mg}$ film-coated tablets; 2007.

28. Deeks ED, Perry CM, Efavirenz/emtricitabine/tenofovir disoproxil fumarate single tablet regimen (Atripla $®$ ): a review of its use in the management of HIV infection. Drugs 2010;70:2315-38.

29. Takahashi M, Kudaka Y, Okumura N, Hirano A, Banno K, Kaneda T. Determination of plasma tenofovir concentrations using a conventional LC-MS method. Biol Pharm Bull 2007;30:1784-6.

30. Llibre JM, Clotet B. Once-daily single-tablet regimens: a long and winding road to excellence in antiretroviral treatment. AIDS Rev 2012;14:168-8.

31. Pedersen OS, Pedersen EB. Non-nucleoside reverse transcriptase inhibitors: the NNRTI boom. Antivir Chem Chemother 1999;10:285-314.

32. Nadal T, Ortuno J, Pascual JA. Rapid and sensitive determination of zidovudine and zidovudine glucuronide in human plasma by ion-pair high-performance liquid chromatography. J Chromatogr A 1996;721:127-37.

33. Food and Drug Administration. Full prescribing information, ATRIPLA $® \quad$ (efavirenz/emtricitabine/tenofovir disoproxil fumarate) tablets. Gilead Sciences; 2013.

34. World Health Organisation, Emtricitabine monograph, 4th ed. The International Pharmacopoiea; 2009.

35. AIDS Info, Emtricitabine; 2014.

36. National Center for Biotechnology Information, Emtricitabine; 2005.

37. Dando TM, Wagstaff AJ. Emtricitabine/tenofovir disoproxil fumarate. Drugs 2004;64:2075-82.

38. Avihingsanon A, Lewin SR, Kerr S, Chang JJ, Piyawat K, Napissanant $\mathrm{N}$, et al. Efficacy of tenofovir disoproxil fumarate/emtricitabine compared with emtricitabine alone in antiretroviral-naive HIV-HBV coinfection in Thailand. Antivir Ther 2010;15:917-22.

39. Moody W. Truvada works to block HIV transmission. Echo Mag 2012;24:66-7.

40. Wang LH, Wiznia AA, Rathore MH, Chittick GE, Bakshi SS, Emmanuel PJ, et al. Pharmacokinetics and safety of single oral doses of emtricitabine in human immunodeficiency virus-infected children. Antimicrob Agents Chemother 2004;48:183-91.

41. Hamarapurkar PD, Parate AN, HPLC method for the determination of emtricitabine and related degradation substances. J Chromatogr Sci 2013;51:419-24.

42. Menendez Arias L. Mechanisms of resistance to nucleoside analogue inhibitors of HIV-1 reverse transcriptase. Virus Res 2008; 134:124-46.

43. Chen A, Dowdy DW. Clinical effectiveness and costeffectiveness of HIV pre exposure prophylaxis in men who have sex with men: risk calculators for real-world decision-making. PLoS One 2014;9:108742.

44. Medicines Control Council, Press release: Medicines Control Council approves fixed-dose combination of tenofovir disoproxyl fumarate and emtricitabine for pre-exposure prophylaxis of HIV. Department of Health; 2015.

45. Varghese V, Wang E, Babrzadeh F, Bachmann MH, Shahriar R, Liu T, et al. Nucleic acid template and the risk of a PCR-Induced HIV-1 drug resistance mutation. PLoS One 2010;5:10992.

46. Boucher S, Recordon Pinson P, Ragnaud JM, Dupon M, Fleury H, Masquelier B. HIV-1 reverse transcriptase (RT) genotypic patterns and treatment characteristics associated with the K65R RT mutation. HIV Med 2006;7:294-8.

47. Gallant JE, Parish MA, Keruly JC, Moore RD. Changes in renal function associated with tenofovir disoproxil fumarate treatment, compared with nucleoside reverse transcriptase inhibitor treatment. Clin Infect Dis Publ Infect Dis Soc Am 2005;40:1194-8.

48. Calza L, Trapani F, Salvadori C, Magistrelli E, Manfredi R, Colangeli $\mathrm{V}$, et al. Incidence of renal toxicity in HIV-infected, antiretroviral-naïve patients starting tenofovir/emtricitabine associated with efavirenz, atazanavir/ritonavir, or lopinavir/ritonavir. Scand J Infect Dis 2013;45:147-54.

49. Deeks SG, Barditch Crovo P, Lietman PS, Hwang F, Cundy KC, Rooney JF, et al. Safety, pharmacokinetics, and antiretroviral activity of 114 intravenous 9-[2-(R)(Phosphonomethoxy)propyl]adenine, a novel anti-human immunodeficiency virus (HIV) therapy, in HIV-infected adults. Antimicrob Agents Chemother 1998;42:2380-4.

50. Chapman T, McGavin J, Noble S. Tenofovir disoproxil fumarate. Drugs 2003;63:1597-608.

51. World Health Organisation, Tenofovir monograph; 2010.

52. AIDS Info, Tenofovir disoproxil fumarate; 2013.

53. Food and Drug Administration, "Full prescribing information, COMPLERA® (emtricitabine/rilpivirine/tenofovir disoproxil fumarate)." Gilead Sciences; 2014.

54. Kearney BP, Flaherty JF, Shah J. Tenofovir disoproxil fumarate: clinical pharmacology and pharmacokinetics. Clin Pharmacokinet 2004;43:595-612.

55. Gervasoni C, Meraviglia P, Landonio S, Baldelli S, Fucile S, Castagnoli $\mathrm{L}$, et al. Low body weight in females is a risk factor for increased tenofovir exposure and drug-related adverse events. PLoS One 2013;8:8-13.

56. Delahunty T, Bushman L, Fletcher CV. Sensitive assay for determining plasma tenofovir concentrations by LC/MS/MS. J Chromatogr B: Anal Technol Biomed Life Sci 2006;830:6-12.

57. Maggiolo F. Efavirenz: a decade of clinical experience in the treatment of HIV. J Antimicrob Chemother 2009;64:910-28.

58. Food and Drug Administration, "Full prescribing information, ATRIPLA ${ }^{\circledR}$ (efavirenz/emtricitabine/tenofovir disoproxil fumarate) tablets." Gilead Sciences; 2013.

59. World Health Organisation, Efavirenz monograph; 2005.

60. AIDS Info, Efavirenz; 2014.

61. Sathigari S, Chadha G, Lee YHP, Wright N, Parsons DL, Rangari $\mathrm{VK}$, et al. Physicochemical characterization of efavirenzcyclodextrin inclusion complexes. AAPS PharmSciTech 2009;10:81-7.

62. McIlleron HM. Effects of rifampin-based antituberculosis therapy on plasma efavirenz concentrations in children vary by CYP2B6 genotype. AIDS 2013;27:1933-40.

63. Friedland G, Khoo S, Jack C, Lalloo U. Administration of efavirenz $[600 \mathrm{mg} /$ day] with rifampicin results in highly variable levels but excellent clinical outcomes in patients treated for tuberculosis and HIV. J Antimicrob Chemother 2006;58:1299-302.

64. Cristofoletti R, Nair A, Abrahamsson B, Groot DW, Kopp S, Langguth $\mathrm{P}$, et al. Biowaiver monographs for immediate release solid oral dosage forms: efavirenz. J Pharm Sci 2013;102:31829.

65. Rabel SR, Patel M, Sun S, Maurin MB. Electronic and resonance effects on the ionization of structural analogues of efavirenz. AAPS Pharm Sci 2001;3:28.

66. AIDS Info, Emtricitabine/tenofovir disoproxil fumarate; 2015.

67. Takagi T, Ramachandran C, Bermejo M, Yamashita S, Yu LX, Amidon GL. A provisional biopharmaceutical classification of the top 200 oral drug products in the United States, Great Britain, Spain, and Japan. Mol Pharm 2006;3:631-43.

68. World Health Organisation. General notes on biopharmaceutics classification system (BCS)-based biowaiver applications; 2011.

69. Drug Bank, Efavirenz; 2013.

70. Drug Bank, Emtricitabine; 2013.

71. Drug Bank, Tenofovir; 2013.

72. Rele RV, Patil SP. Development of analytical method by RPHPLC method for validation of emtricitabine in api and pharmaceutical dosage form. Asian J Res Chem 2019;12:143-7.

73. Singh G, Pai RS. Optimization (Central Composite Design) and validation of HPLC method for investigation of emtricitabine loaded poly (lactic-co-glycolic acid) nanoparticles: in vitro drug release and in vivo pharmacokinetic studies. Sci World J 2014. DOI:10.1155/2014/583090

74. Singh G, Pai RS. High-performance liquid chromatographic method for analysis of emtricitabine in rat plasma: method development, validation and application to a pharmacokinetic study. ISRN Chromatography 2013;3:1-16. 
75. Kumar P, Dwivedi SC, Kushnoor A. A validated stability indicating RPHPLC method for the determination of emtricitabine in bulk and capsules. Farmacia 2012;60:402-10.

76. Patel BN, Suhagia BN, Patel CN. Development and validation of HPLC method for the estimation of emtricitabine in capsule dosage form. Asian J Chem 2010;3:869-71.

77. Jampala RR, Kumar VK, Nemala AR. Development and application of liquid chromatographic method for simultaneous determination of elvitegravir, tenofovir disoproxil fumarate, emtricitabine, and cobicistat in fixed dosage form. Pharm Methods 2014;5:7-13.

78. Venkatesh M, Sumanth ALMN, Rao PV. Analytical method development and validation of simultenious estimation of tenofovir and emtricitabine in bulk and pharmaceutical dosageforms by using RP-HPLC. Asian J Pharm Anal Med Chem 2013;1:60-9.

79. Kiran KK, Rao AS, Sankar DG. New validated, optimized and forced degradation study for the simultaneous estimation of rilpivirine, emtricitabine, and tenofovir alafenamide in bulk and pharmaceutical dosage preparations by RP-HPLC. Asian J Pharm Anal Med Chem 2017;5:170-87.

80. Sattar MDA, Achanta S. Analytical method development and validation for the determination of emtricitabine and tenofovir disoproxil fumarate using reverse phase HPLC method in bulk and tablet dosage form. J Pharm Sci Res 2018;10:1207-12.

81. Akram NMD, Umamahesh M. A new validated RP-HPLC method for the determination of emtricitabine and tenofovir af in its bulk and pharmaceutical dosage forms. J Chem Pharm Sci 2017;10:54-9.

82. Ashok G, Mondal S. Development and validation of stability indicating method for the simultaneous quantification of emtricitabine, tenofovir disoproxil fumarate and rilpivirine hydrochloride in pharmaceutical dosage forms by RP-HPLC. Saudi J Med Pharm Sci 2018;4:175-83.

83. Gummaluri RK, Parthasarathi TVN, Anjanamadhulika G. Simultaneous method for determination of emtricitabine, tenofovir disoproxil fumarate, elvitegravir and cobicistat in tablets by HPLC. Indian J Pharm Sci 2016;78:532-7.

84. Soni A, Thakral S. Simultaneous estimation of tenofovir and emtricitabine in human plasma using HPLC after protein precipitation extraction. J Anal Bioanal Tech 2013;4:170.

85. Pranitha D, Vanitha C, Francis P, Raja MA, Vardan PV, Surendar $M$, et al. Simultaneous estimation of emtricitabine, tenofovir disoproxil fumarate, and rilpivirine in bulk form by RP-HPLC method. J Pharm Res 2012;5:4600-2.

86. Gopal NM, Sridhar C. Reverse phase UPLC method for simultaneous estimation of emtricitabine and tenofovir in tablet dosage form. J Sci Res Pharm 2017;6:11-7.

87. Basha A, Sireesha D, Anil D, Talla R, Haque MA, Harshini S, et al. Method development and validation for simultaneous estimation of tenofovir disoproxil fumarate and emtricitabine in pharmaceutical dosage form by RP-HPLC method. Int J Innovative Pharm Sci Res 2015;3:1537-45.

88. Muthyala S, Kumari RVV. Stability indicating RP-HPLC method for simultaneous estimation of emtricitabine, bictegravir and tenofovir alafenamide in bulk and formulation. Int J Pharm Anal Res 2019;8:281-92.

89. Reddy BRY, Reddy MT, Reddy BSC. Simultaneous estimation of emtricitabine and tenofovir disoproxil fumarate in tablet dosage form by reverse phase high-performance liquid chromatography. SOJ Chromatograph Sci 2015;1:6.

90. Runja C, Kumar PR, Avanapu SR. A validated stability indicating RP-HPLC method for the determination of emtricitabine, tenofovir disoproxil fumarate, elvitegravir and cobicistat in pharmaceutical dosage form. J Chromatographic Sci 2016;54:759-64.

91. Karunakaran AK, Kamarajan K, Thangarasu V. A validated RPHPLC method for simulataneous estimation of emtricitabine and tenofovir disoproxil fumarate in pure and in tablet dosage form. Pharm Sinica 2010;1:52-60.

92. Purnima BV, Reddy TVB, Rao YS, Ramu G, Ramachandran D. Stability indicating RP-UPLC method for assay of emtricitabine and tenofovir disoproxil fumarate in bulk and dosage forms. Am J Anal Chem 2015;6:807-21.
93. Sharma R, Gupta P. A validated RP-HPLC method for simulataneous estimation of emtricitabine and tenofovir disoproxil fumarate in a tablet dosage form. Eurasian J Anal Chem 2009;4:276-84.

94. Joshi NC, Kumar P, Jat RK. Development and validation of RPHPLC method for the estimation of antiretroviral drugs and their pharmaceutical formulations. Pharmacophore 2016;7:152-65

95. Purnima VB, Reddy TVB, Suneetha Y, Ramachandran D. Simultaneous determination of antiretroviral drugs emtricitabine and tenofovir desoproxil fumerate by a stability indicating RP-HPLC method. Int $\mathrm{J}$ Pharm Pharm Sci 2015;7:390-8.

96. Khaleel N, Rahaman SA. A validated stability indicating RPHPLC method for simultaneous estimation of tenofovir disoproxil fumarate, cobicistat, emtricitabine and elvitegravir in bulk and pharmaceutical dosage form. Int J Pharm 2015;5:991-1002.

97. Komaroju D, Reddy GN, Dhanalakshmi K. Method development and validation for simultaneous estimation of emtricitabine and tenofovir disoproxil fumarate in pure and tablet dosage form by using RP-HPLC. Int J Pharma Res Rev 2013;2:1-11.

98. Jagadabi V, Kumar PVN, Pamidi S, Ramaprasad LA, Pavani G. Identification and quantification of potential impurities using LC-PDA coupled with new qda mass detector in a new single tablet regimen containing dolutegravir, emtricitabine and tenofovir disoproxil fumarate tablets used in hiv-1 prevention. Int Res J Pharm 2019;10:91-104.

99. Kuna M, Dannana GS, Prasad SVUM. Development and validation of a new RP-HPLC method for simultaneous estimation of emtricitabine, tenofovir, cobicistat, elvitegravir and its comparision with a reported method. Indo Am J Pharm Sci 2017;4:1599-612.

100. Nagasarapu MR, Dannana GS. Development and validation of stability-indicating HPLC-DAD method for simultaneous determination of emtricitabine, elvetegravir, cobicistat and tenofovir in their tablet dosage forms. Indian J Pharm Edu Res 2016;50:205-11.

101. Saidulu P, Mastanamma SK, Suresh PV, Rani AP. Development and validation of stability-indicating HPLC-DAD method for simultaneous determination of emtricitabine, rilpivirine, and tenofovir alafenamide in bulk and their pharmaceutical dosage forms. Int J ChemTech Res 2018;11:329-39.

102. Nandini K, Muneer S, Sekhar KBC, Kiran BSS. Stability indicating RP-HPLC method development and validation for the quantification of tenofovir disoproxil fumarate in bulk and its dosage form. Int J PharmTech Res 2016;9:240-9.

103. Dubbaka A, Sireesha D, Bakshi V. Analytical method development and validation for the simultaneous estimation of lamivudine and tenofovir disoproxil fumarate by RP-HPLC method. MOJ Proteomics Bioinform 2016;4:306-9.

104. Sonawane PH, Panzade PS, Kale MA. Simultaneous estimation of lamivudine and tenofovir disoproxil fumarate in bulk and combined pharmaceutical dosage form by HPLC method. Asian J Biomed Pharm Sci 2013;3:27-30.

105. Saha C, Gupta NV, Chandan RS, Priya SP. Development of a validated stability indicating LC-MS method for the determination of tenofovir disoproxil fumarate using quality by design approach. Int J Appl Pharm 2019;11:406-17.

106. Babu C, Devanna N, Reddy KVNS. Validated gradient stability indicating RP-HPLC method for the simultaneous quantification of 11 related substances in the combined dosage forms of lamivudine and tenofovir disopeoxil fumarate. Int J Appl Pharm 2017;9:61-8.

107. Diana BH, Bibi SK, Kumari KS. New validated RP-HPLC method for simultaneous estimation of lamivudine and tenofovir disproxil fumarate in tablets. Int J Adv Pharm Anal 2015;5:10-3.

108. Havele S, Dhaneshwar SR. Development and validation of a stability-indicating LC method for the determination of tenofovir disoproxil fumarate in pharmaceutical formulation. Songklanakarin J Sci Technol 2012;34:615-22.

109. Ravisankar P, Mounika G, Devadasu CH, Rao GD. Novel analytical method development and validation for the 
quantitative analysis of efavirenz in bulk and pharmaceutical dosage forms by RP-HPLC. Pharma Innovation J 2014;3:32-9.

110. Gupta S, Kesarla R, Chotai N, Omri A. Development and validation of reversed phase HPLC gradient method for the estimation of efavirenz in plasma. PLoS One 2017;12:0174777.

111. Suneetha A, Bhavana G, Siddikha ASK. Development and validation of stability indicating RP-HPLC method for simultaneous estimation of efavirenz, lamivudine, and stavudine in pharmaceutical dosage forms. BAOJ Pharm Sci 2017;3:41.

112. Viana OS, Medeiros FPM, Grangeiro Junior S, Albuquerque MM Soares MFR. Development and validation of a HPLC analytical assay method for efavirenz tablets: a medicine for HIV infections. Brazilian J Pharm Sci 2011;47:97-102.

113. Kumar GS, Patnaik P, Patnaik A, Subrahmanyam KV. A new method development and validation of efavirenz by RP-HPLC. Int J Innovative Pharm Sci Res 2014;2:2962-70.

114. Waghmare SA, Kashid AM. Reverse phase-high performance liquid chromatography method development and validation for estimation of efavirenz by quality by design approach. J Drug Delivery Ther 2019;9:319-30.

115. Induri M, Mantripragada BR, Yejella RP. Development and validation of UPLC method for simultaneous estimation of Efavirenz and Lamivudine in pharmaceutical formulations. J Appl Pharm Sci 2016;6:29-33.

116. Bhavsar DS, Patel BN, Patel CN. RP-HPLC method for simultaneous estimation of tenofovir disoproxil fumarate, lamivudine, and efavirenz in combined tablet dosage form. Pharmaceutical Methods 2012;3:73-8.

117. Panchagiri S, Begum A, Valupadas C, Ciddi V. Quantitative bioanalysis of tenofovir disoproxil fumarate, lamivudine and efavirenz simultaneously in human plasma using reverse-phase liquid chromatography. Acta Sci Pharm Sci 2018;2:17-27.

118. Sumanth KS, Rao AS, Shankar DG. A new gradient RP-HPLC method development and validation for simultaneous estimation of lamivudine, tenofovir disoproxil fumarate and effavirenz in pharmaceutical dosage forms. Int J Pharm Chem Biol Sci 2018;8:195-203.

119. Maniyar UR, Koshe K, Katariya MV, Karva GS, Katariya VR Jaiswal S. Stability indicating RP-HPLC method development and validation for the determination of potential degradation impurities of efavirenz, emtricitabine and tenofovir in combined pharmaceutical dosage form. Asian J Pharm Technol Innovation 2015;3:117-28

120. Raju NA, Begum S. Simultaneous RP-HPLC method for the estimation of the emtricitabine, tenofovir disoproxil fumerate and efavirenz in tablet dosage forms. Res J Pharm Tech 2008;1:522-5

121. Nirogi R, Bhyrapuneni G, Kandikere V, Mudigonda K, Komarneni P, Aleti R, et al. Simultaneous quantification of a non-nucleoside reverse transcriptase inhibitor efavirenz, a nucleoside reverse transcriptase inhibitor emtricitabine and a nucleotide reverse transcriptase inhibitor tenofovir in plasma by liquid chromatography positive ion electrospray tandem mass spectrometry. Biomed Chromatogr 2009;23:371-81.

122. Babu C, Rao LNK, Devanna N, Reddy SKVN. RP-UPLC method for the simultaneous quantification of related substances in emtricitabine, tenofovir disoproxil fumarate and efavirenz pharmaceutical dosage forms. J Chem Pharm Res 2017;9:45-52.

123. Rezaei M, Ramazani A, Hokmabadi F. Simultaneous estimation and validation of tenofovir disoproxil fumarate, emtricitabine and efavirenz by RP-HPLC method in combined tablet dosage form. Curr Pharm Anal 2019;15:561-7.

124. Sreelatha P, Devi BR. Development and validation of stabilityindicating HPLC method for simultaneous estimation of tenofovir, emtricitabine and efavirenz in fixed dose combination drug product. Asian J Res Chem 2018;11:23-31.

125. Kumar KB, Ayele Y, Motbatnor B. A novel validated stability indacating method for simultaneous estimation of emtricitabine, tenofovir and efavirenz in tablet dosage form by RP-HPLC. Int J Med Pharm Res 2019;7:71-82.

126. Varma PSRCHNPD, Rao AL. Stability-indicating RP-HPLC method for the simultaneous estimation of efavirenz, tenofovir and emtricitabine in pharmaceutical formulations. Indian J Pharm Pharmacol 2014;1:1-19.
127. Tiwari P, Yadav R, Avinash KV, Vaidya PA, Sathe, Gangrade D. Development and validation of UPLC method for emtricitabine, tenofovir and efavirenz in pharmaceutical preparation. Anal Chem Indian J 2010;9:247-51.

128. Ashenafi D, Ungerbock M, Hoogmartens J, Adams E. Liquid chromatographic analysis of various formulations containing emtricitabine. Chromatographia 2013;76:1495-503.

129. Rao SA, Kumar NG, Srilekha K, Kumari AN. Stability indicating method for the simultaneous estimation of tenofovir emtricitabine and efavirenz in pure and pharmaceutical dosage form by RP-HPLC. Int J Adv Res Sci Eng 2016;5:188-200.

130. Devrukhakar PS, Borkar R, Shastri N, Surendranath KV. A validated stability-indicating RP-HPLC method for the simultaneous determination of tenofovir, emtricitabine, and an efavirenz and statistical approach to determine the effect of $\begin{array}{llll}\text { variables. } & \text { ISRN } & \text { Chromatography } & 2013 .\end{array}$ https://doi.org/10.1155/2013/878295

131. Ramaswamy A, Arul AS, Dhas G. Development and validation of analytical method for quantitation of emtricitabine, tenofovir efavirenz based on HPLC. Arabian J Chem 2018;11:275-81.

132. Sravanthi T, Madhavi N. Stability indicating UPLC method to quantify emtricitabine, tenofovir, and efavirenz simultaneously in tablets: method establishment. Int J Res Pharm Sci 2020;11:120-8

133. Nadig S, Jacob JT, Bhat I, Kishoreraju V. A stability indicating RP-HPLC method for simultaneous estimation of Emtricitabine, Tenofovir disoproxil fumarate and Efavirenz in pharmaceutical dosage forms. Int J Res Pharm Sci 2013;4:391-6.

134. Badgujar BP, Mahajan MP, Sawant SD. Development and validation of RP-HPLC method for the simultaneous estimation of tenofovir alafenamide and emtricitabine in bulk and tablet dosage form. Int J Chem Tech Res 2017;10:731-9.

135. Panigrahy UP, Reddy AS. A novel validated RP-HPLC method for the simultaneous estimation of emtricitabine, tenofovir disoproxil fumarate and rilpivirine in bulk and pharmaceutical tablet dosage forms. Pharm Lett 2015;7:303-14.

136. Sayyed ZM, Sheikh AA, Shaikh ZA, Shinde SA. Development and validation of analytical method for simultaneous estimation of tenofovir and emtricitabine in pharmaceutical dosage forms by HPLC. Int J Pharm Drug Anal 2016;4:24-9.

137. Kavitha KY, Geetha G, Hariprasad R, Venkatnarayana R, Kaviarasu M. Development and validation of RP-UPLC analytical method for simultaneous estimation of emtricitabine, rilpivirine, tenofovir disoproxil fumarate and its pharmaceutical dosage forms. Int Res J Pharm 2013;4:150-5.

138. Yadav M, Singhal P, Goswami S, Pande UC, Sanyal M, Shrivastav PS. Selective determination of antiretroviral agents tenofovir, emtricitabine, and lamivudine in human Plasma by a LC-MS-MS method for a bioequivalence study in healthy Indian subjects. J Chromatographic Sci 2010;48:704-13.

139. Ahmad S, Rageeb M, Usman M. Analytical method development and validation for the simultaneous estimation of emtricitabine and tenofovir by reversed-phase high performance liquid chromatography in bulk and tablet dosage forms. Asian J Pharm Clin Res 2017;10:59-64.

140. Abdelhay MH, Gazy AA, Shaalan RA, Ashour HK. Selective RPHPLC dad method for determination of tenofovir fumarate and emtricitabine in bulk powder and in tablets. Acta Chromatographica 2015;27:41-54.

141. Ashenafi D, Verbeek A, Hoogmartens J, Adams E. Development and validation of an LC method for the determination of emtricitabine and related compounds in the drug substance. J Sep Sci 2009;32:1823-30.

142. Delahunty T, Bushman L, Robbins B, Fletcher CV. The simultaneous assay of tenofovir and emtricitabine in plasma using LC/MS/MS and isotopically labeled internal standards. J Chromatography B 2009;877:1907-14.

143. Gomes NA, Vaidya VV, Pudage A, Joshi SS, Parekh SA. Liquid chromatography-tandem mass spectrometry (LC-MS/MS) method for simultaneous determination of tenofovir and emtricitabine in human plasma and its application to a bioequivalence study. J Pharm Biomed Anal 2008;48:918-26.

144. Illamola SM, Valade E, Hirt D, Dulioust E, Zhengb Y, Wolfd JP, et al. Development and validation of a LC-MS/MS method for the 
quantification of tenofovir and emtricitabine in seminal plasma. J Chromatography B 2016;1033:234-41.

145. Venkateswararao B, Vidyadhara S, Nagaraju B, Jhonbi SK. A novel stability indicating RP-HPLC method development and validation for the determination of tenofovirdisoproxilfumarate and emtricitabine in bulk and pharmaceutical formulations. Int J Pharm Sci Res 2017;8:2168-76.

146. Mastanamma SK, Reddy VD, Saidulu P, Varalakhimi M. Development and validation of stability indicating RP-HPLC method for the simultaneous estimation of emtricitabine tenofovir alafenamide bulk and their combined dosage form. J Chem Pharm Res 2017;9:70-80.

147. Raju NA, Rao JV, Prakash KV, Mukkanti K, Srinivasu K. Simultaneous estimation of tenofovir disoproxil, emtricitabine and efavirenz in tablet dosage form by RP-HPLC. Orient J Chem 2008;24:645-50.

148. Cholke P, Chemate SZ, Joshi RS, Raskar MA, Sawant RL. Development and validation of spectrophotometric method for efavirenz in pure and in film coated tablet dosage form. Res J Pharm Technol 2011;4:1816-8.

149. Kumar P, Mittan DS, Kumar N. Development of UV spectroscopic method for the estimation of tenofovir in bulk and solid dosage forms. Anal Chem Indian J 2013;13:33-5.

150. Vidyadhara S, Sasidhar RLC, Rao BV, Kumari PR. Simultaneous UV spectrophotometric method for the determination of tenofovir, efavirenz and lamivudine in bulk and combined dosage form. Asian J Pharm Anal 2016;6:253-8.

151. Rajan RV. UV spectrophotometric estimation of emtricitabine by zero order and area under curve methods in bulk and pharmaceutical dosage form. Asian J Res Chem 2019;12:263-7.

152. Rajavel P. Analytical method development and validation of tenofovir by UV spectrophotometry. Asian J Res Pharm Sci Biotechnol 2016;4:88-94.

153. Srinath A, Sneha B, Alladi A, Ahmed R, Kulkarni RG. Method development and validation for simultaneous estimation of lamivudine, tenofovir and efavirenz in combined tablet dosage form by RP-HPLC and UV-spectroscopic method. Int J Pharm Sci Res 2014;5:5491-7.

154. Shelke A, Shinde M, Mogal R, Sable R, Jadhav A. Application of UV spectrophotometric methods for simultaneous estimation of emtricitabine and tenofovir alafenamide fumarate in bulk. Asian J Pharm Tech 2018;8:103-7.

155. Lavanya B, Hariprasad P, Venkatapraveen A, lakshmi DP, Dhanalakshmi. Method development and validation of combined tablet dosage form of emtricitabine and tenofovir disproxil fumerate by ultraviolet spectroscopy. Int Res J Pharm 2012;3:104-8.

156. Anandakumar K, Kannan K, Vetrichelvan T. Development and validation of emtricitabine and tenofovir disoproxil fumerate in pure and in fixed dose combination by uv spectrophotometry. Digest J Nanomaterials Biostructures 2011;6:1085-90.

157. Sri KV, Yadla DK, Shalem M, Sirisha VRK. Simultaneous estimation and validation of emtricitabine, tenofovir disproxil fumarate and efavirenz in pharmaceutical dosage form by UVspectrophotometry. Chem Sci Rev Lett 2017;6:2581-9.

158. Harini U, Pawar AKM. Development and validation of stability indicating simultaneous uvspectrophotometric method for determination of emtricitabine, tenofovir disoproxil fumarate, cobicistat, and elvitegravir in pure and pharmaceutical dosage form. Asian J Pharm Clin Res 2018;11:177-84.

159. Nagaraju PT, Channabasavaraj KP, Shantha KPT. Development and validation of spectrophotometric method for estimation of emtricitabine in tablet dosage form. Int $\mathrm{J}$ ChemTech Res 2011;3:23-8.

160. Behera A, Parida A, Meher AK, Dannana GS, Moitra SK, Chandra SS. Development and validation of spectrophotometric method for determination of emtricitabine and tenofovir disoproxil fumarate in bulk and tablet dosage form. Int J PharmTech Res 2011;3:1874-82.

161. Shirkhedkar AA, Bhirud CH, Surana SJ. Application of uvspectrophotometric methods for estimation of tenofovir disoproxil fumarate in tablets. Pak J Pharm Sci 2009;22:27-9.

162. Sharma R, Mehta K. Simultaneous spectrophotometric estimation of tenofovir disoproxil fumarate and lamivudine in three component tablet formulation containing efavirenz. Indian J Pharm Sci 2010;72:527-30.

163. Venkatesan S, Kannappan N. Simultaneous spectrophotometric method for determination of emtricitabine and tenofovir disoproxil fumarate in three-component tablet formulation containing rilpivirine hydrochloride. Int Scholarly Res Notices 2014. https://doi.org/10.1155/2014/541727

164. Ingale KD, Barhate AL, Kale AN, Bobade CD, Choudhari VP Kuchekar BS. Spectrophotometric estimation of emtricitabine and tenofovir disoproxil fumarate in tablet dosage form by simultaneous equation and absorbance ratio methods. J Pharm Res 2010;9:11-3

165. Choudhari VP, Ingale S, Gite SR, Tajane DD, Modak VG, Ambekar A. Spectrophotometric simultaneous determination of tenofovir disoproxil fumarate and emtricitabine in combined tablet dosage form by ratio derivative, first order derivative and absorbance corrected methods and its application to dissolution study. Pharm Methods 2011;2:47-52.

166. Ashour HK, Belal TS. New simple spectrophotometric method for determination of the antiviral mixture of emtricitabine and tenofovir Disoproxilfumarate. Arabian J Chem 2017;10:1741-7.

167. Dubbaka A, Sireesha D, Basha SA, Talla R, Haque MA, Harshini $\mathrm{S}$, et al. Analytical method development and validation for the simultaneous estimation of lamivudine and tenofovir disoproxil fumarate in bulk and spectrophotometric method. Int J Innovative Pharm Sci Res 2015;3:1529-36.

168. Induri M, Mantripragada BR, Yejella RP. Development and validation of a HPTLC method for simultaneous estimation of efavirenz and lamivudine in pharmaceutical formulations. World J Pharm Sci 2016;4:189-94.

169. Pratima NA, Ajhar M, Shaikh O. HPTLC method development, validation for simultaneous determination of efavirenz, emtricitabine and tenofovir in combined tablet formulation and forced degradation studies. Am J PharmTech Res 2013;3:65060.

170. Chitlange SS, Kanthale SB, Choudhary B, Bhole RP. Stability indicating HPTLC method for the simultaneous estimation of rilpivirin, emtricitabine and tenofovir in bulk and combined pharmaceutical dosage form. J Adv Drug Delivery 2014;1:15772.

171. Chandra P, Rathore AS, Sathiyanarayanan L, Mahadik KR. Application of high performance thin-layer chromatographic method for the simultaneous determination of lamivudine and tenofovir disoproxil fumarate in pharmaceutical dosage form. J Chil Chem Soc 2011;56:702-5.

172. Kumar P, Dwivedi SC, Kushnoor A. Development and validation of HPTLC method for the determination of Efavirenz in bulk drug and tablet dosage form. Res J Pharm Biol Chem Sci 2011;2:160-8

173. Shinde NV, Tompe PU, Damle MC, Madgulkar AR. Development and validation of HPTLC method for estimation of efavirenz in human plasma. J Chem Pharm Res 2013;5:85-90.

174. Mardia RB, Suhagia BN, Pasha TY, Chauhan SP, Solanki S. Development and validation of HPTLC method for estimation of tenofovir disoproxil fumarate in tablet dosage form. J Pharm Sci Biosci Res 2012;2:73-6.

175. Saminathan J, Vetrichelvan T. Development and validation of HPTLC method for simultaneous estimation of emtricitabine, rilpivirine and tenofovir disoproxil fumarate in combined dosage form. Bangladesh Pharm J 2016;19:114-21. 